\title{
ALGEBRAS OF ACYCLIC CLUSTER TYPE: TREE TYPE AND TYPE $\widetilde{A}$
}

\author{
CLAIRE AMIOT AND STEFFEN OPPERMANN
}

\begin{abstract}
In this paper, we study algebras of global dimension at most 2 whose generalized cluster category is equivalent to the cluster category of an acyclic quiver which is either a tree or of type $\widetilde{A}$. We are particularly interested in their derived equivalence classification. We prove that each algebra which is cluster equivalent to a tree quiver is derived equivalent to the path algebra of this tree. Then we describe explicitly the algebras of cluster type $\widetilde{A}_{n}$ for each possible orientation of $\widetilde{A}_{n}$. We give an explicit way to read off the derived equivalence class in which such an algebra lies, and we describe the Auslander-Reiten quiver of its derived category. Together, these results in particular provide a complete classification of algebras which are cluster equivalent to tame acyclic quivers.
\end{abstract}

\section{$\S 1$. Introduction}

The classification of finite-dimensional algebras over an algebraically closed field $k$ up to derived equivalence is a crucial problem in representation theory. It has a complete answer for algebras of global dimension 1 (see [H, Corollary 4.8]): two finite-dimensional $k$-algebras $\Lambda=k Q$ and $\Lambda^{\prime}=k Q^{\prime}$ are derived equivalent if and only if one can pass from the quiver $Q$ to the quiver $Q^{\prime}$ by a sequence of reflections (as introduced in [BGP]). Therefore, it is possible to decide when two hereditary algebras are derived equivalent by simple combinatorial means. The aim of this paper is to apply results of [AO1] in order to generalize this result to certain algebras of global dimension 2 .

Received September 24, 2011. Revised March 9, 2012. Accepted May 10, 2012.

First published online April 26, 2013.

2010 Mathematics Subject Classification. Primary 16E35, 16G20; Secondary 16G70, $16 \mathrm{E} 10,16 \mathrm{~W} 50$.

Both authors' work was partially supported by Norwegian Research Council Storforsk grant 167130. Amiot's work was partially supported by Agence Nationale de la Recherche project ANR-09-BLAN-0039-02.

(C) 2013 by The Editorial Board of the Nagoya Mathematical Journal 
The notion of reflection of an acyclic quiver has been generalized by Fomin and Zelevinsky [FZ] to the notion of mutation in their definition of cluster algebras. Since then, categorical interpretations of the mutation have been discovered via 2-Calabi-Yau triangulated categories. These created a link between cluster algebras and representation theory. First, in [BMRRT, Section 1], cluster categories $\mathcal{C}_{Q}$ associated to acyclic quivers $Q$ were defined as the orbit categories $\mathcal{D}^{b}(k Q) / \mathbb{S}_{2}$, where $\mathbb{S}_{2}=\mathbb{S}[-2]$ is the second desuspension of the Serre functor $\mathbb{S}$ of the bounded derived category $\mathcal{D}^{b}(k Q)$. This notion has been generalized in $[\mathrm{A}]$ to algebras of global dimension 2 . In this case, the generalized cluster category is defined to be the triangulated hull in the sense of $[\mathrm{K} 2]$ of the orbit category $\mathcal{D}^{b} \Lambda / \mathbb{S}_{2}$.

In this article, we study more explicitly the derived equivalence classes of algebras of global dimension 2 which are of acyclic cluster type, that is, algebras whose generalized cluster category is equivalent to some cluster category $\mathcal{C}_{Q}$, where $Q$ is an acyclic quiver. We use extensively the results and the techniques of [AO1]. In particular, we use the notion of graded mutation of a graded quiver with potential (graded QP), which is a refinement of the notion of mutation of a quiver with potential introduced in [DWZ]: associated to an algebra $\Lambda$, there is a graded Jacobian algebra $\bar{\Lambda}$ (see [K3, Section 6.11]) whose degree 0 subalgebra is $\Lambda$. Graded mutation explains how to mutate such graded Jacobian algebras. Then, from [AO1] we deduce an analogue of the result for algebras of global dimension 1.

TheOrem 1.1 (see Theorem 3.15). Let $\Lambda_{1}$ and $\Lambda_{2}$ be two finite-dimensional algebras of global dimension 2. Assume that $\Lambda_{1}$ is of acyclic cluster type. Then the algebras $\Lambda_{1}$ and $\Lambda_{2}$ are derived equivalent if and only if one can pass from $\bar{\Lambda}_{1}$ to $\bar{\Lambda}_{2}$ using a sequence of graded mutations.

The setup is especially nice when the algebras are of tree-cluster type.

THEOREM 1.2 (see Corollary 3.16). Let $Q$ be an acyclic quiver whose underlying graph is a tree. If $\Lambda$ is an algebra of global dimension 2 of cluster type $Q$, then it is derived equivalent to $k Q$.

To get a complete understanding of algebras of tame acyclic cluster type, in the rest of this paper we focus on the algebras of cluster type $\widetilde{A}_{p, q}$. To such an algebra, using graded mutation, we associate an integer that we call weight, which is zero when $\Lambda$ is hereditary. We prove that two algebras of cluster type $\widetilde{A}_{p, q}$ are derived equivalent if and only if they have the same weight (Theorem 4.5). Then a result of [AO1, Theorem 5.6], which shows 
that two cluster equivalent algebras are graded derived equivalent, permits us to compute explicitly the shape of the Auslander-Reiten quiver of the derived category.

THEOREM 1.3 (see Corollary 5.5). Let $\Lambda$ be an algebra of cluster type $\widetilde{A}_{p, q}$ and of weight $w \neq 0$. Then the algebra $\Lambda$ is representation-finite and not piecewise hereditary. The Auslander-Reiten quiver of $\mathcal{D}^{b}(\Lambda)$ has exactly $3|w|$ connected components:

- $|w|$ components of type $\mathbb{Z} A_{\infty}^{\infty}$,

- $2|w|$ components of type $\mathbb{Z} A_{\infty}$.

Finally, we use the explicit description of the cluster-tilted algebras of type $\widetilde{A}_{p, q}$ of $[\mathrm{Ba}]$ to deduce a description of all algebras of cluster type $\widetilde{A}_{p, q}$ in terms of quivers with relations.

This article is organized as follows. Section 2 is devoted to recalling results on generalized cluster categories, on Jacobian algebras, and on 2-Calabi-Yau categories. In Section 3, we apply the results of [AO1] to algebras of acyclic cluster type. The special case of algebras of cluster type $\widetilde{A}_{p, q}$ is treated in Sections 4-6. We introduce the notion of weight and prove that it is an invariant of the derived equivalence class of the algebra in Section 4. We compute the shape of the Auslander-Reiten quiver of the derived category in Section 5, and we describe these algebras explicitly in Section 6. We end the paper by giving as an example the complete classification for $\widetilde{A}_{2,2}$.

\section{$\S 2$. Background}

Throughout this paper, $k$ denotes an algebraically closed field. All categories appearing are $k$-categories, and all functors are $k$-linear. By an algebra we mean an associative unitary basic $k$-algebra. For an algebra $\Lambda$, we denote by $\bmod \Lambda$ the category of finitely generated right modules over $\Lambda$. We denote by $D$ the standard duality $\operatorname{Hom}_{k}(-, k):(\bmod k)^{\mathrm{op}} \rightarrow \bmod k$.

\subsection{Cluster-tilting objects and mutation in 2-Calabi-Yau cat- egories}

Definition 2.1. Let $\mathcal{T}$ be a Krull-Schmidt triangulated category, with finite-dimensional Hom-spaces (Hom-finite for short) and 2-Calabi-Yau; that is, there is a functorial isomorphism $\operatorname{Hom}_{\mathcal{T}}(X, Y[2]) \cong D \operatorname{Hom}_{\mathcal{T}}(Y, X)$ for all objects $X$ and $Y$ in $\mathcal{T}$. An object $T$ is called cluster tilting if

$$
\operatorname{add}(T)=\left\{X \in \mathcal{T} \mid \operatorname{Hom}_{\mathcal{T}}(X, T[1])=0\right\},
$$

where $\operatorname{add}(T) \subset \mathcal{T}$ is the additive closure of $T$. 
The endomorphism algebra of a cluster-tilting object $T \in \mathcal{T}$ is called a 2-Calabi-Yau-tilted algebra.

TheOREM 2.2 ([IY, Theorem 5.3]). Let $T$ be a basic cluster-tilting object in a 2-Calabi-Yau triangulated category $\mathcal{T}$. Let $T_{i}$ be an indecomposable summand of $T \cong T_{i} \oplus T^{\prime}$. Then there exists a unique (up to isomorphism) object $T_{i}^{*}$ not isomorphic to $T_{i}$, such that $\mu_{T_{i}}(T):=T^{\prime} \oplus T_{i}^{*}$ is a basic clustertilting object in $\mathcal{T}$. Moreover, $T_{i}^{*}$ is indecomposable, and there exist triangles in $\mathcal{T}$

$$
T_{i} \stackrel{u}{\longrightarrow} U \longrightarrow T_{i}^{*} \longrightarrow T_{i}[1]
$$

and

$$
T_{i}^{*} \longrightarrow U^{\prime} \stackrel{u^{\prime}}{\longrightarrow} T_{i} \longrightarrow T_{i}^{*}[1],
$$

where $u$ (resp., $\left.u^{\prime}\right)$ is a minimal left (resp., right) add $\left(T^{\prime}\right)$-approximation.

\subsection{Generalized cluster categories}

Let $\Lambda$ be a finite-dimensional $k$-algebra of global dimension at most 2 . We denote by $\mathcal{D}^{b}(\Lambda)$ the bounded derived category of finitely generated $\Lambda$ modules. It has a Serre functor that we denote by $\mathbb{S}$. We denote by $\mathbb{S}_{2}$ the composition $\mathbb{S}[-2]$.

The generalized cluster category $\mathcal{C}_{\Lambda}$ of $\Lambda$ has been defined in [A, Section 4] as the triangulated hull of the orbit category $\mathcal{D}^{b}(\Lambda) / \mathbb{S}_{2}$ (see $[\mathrm{K} 2]$ or $[\mathrm{AO} 1$, Section 7] for more details on triangulated hulls). We will denote by $\pi_{\Lambda}$ the triangle functor

$$
\pi_{\Lambda}: \mathcal{D}^{b}(\Lambda) \longrightarrow \mathcal{D}^{b}(\Lambda) / \mathbb{S}_{2} \longrightarrow \mathcal{C}_{\Lambda}
$$

We set $\bar{\Lambda}:=\operatorname{End}_{\mathcal{C}}(\pi \Lambda)=\bigoplus_{p \in \mathbb{Z}} \operatorname{Hom}_{\mathcal{D}}\left(\Lambda, \mathbb{S}_{2}^{-p} \Lambda\right)$. By definition, this algebra is naturally endowed with a $\mathbb{Z}$-grading.

If $\Lambda=k Q$ is the path algebra of an acyclic quiver, then the cluster category $\mathcal{C}_{\Lambda}=\mathcal{C}_{Q}$ has been introduced in [BMRRT, Section 1]; in this article we call it the acyclic cluster category.

Definition 2.3. A finite-dimensional $k$-algebra $\Lambda$ of global dimension at most 2 is said to be $\tau_{2}$-finite if the algebra $\operatorname{End}_{\mathcal{C}}(\pi \Lambda)=\bigoplus_{p \in \mathbb{Z}} \operatorname{Hom}_{\mathcal{D}}\left(\Lambda, \mathbb{S}_{2}^{-p} \Lambda\right)$ is finite-dimensional.

TheOREM 2.4 ([A, Theorem 4.10]). Let $\Lambda$ be a finite-dimensional algebra of global dimension at most 2 which is $\tau_{2}$-finite. Then $\mathcal{C}_{\Lambda}$ is a Hom-finite, 2-Calabi-Yau category, and the object $\pi(\Lambda) \in \mathcal{C}_{\Lambda}$ is a cluster-tilting object. 


\subsection{Jacobian algebras and 2-Calabi-Yau-tilted algebras}

Quivers with potentials and the associated Jacobian algebras have been studied in [DWZ]. Let $Q$ be a finite quiver. For each arrow $a$ in $Q$, the cyclic derivative $\partial_{a}$ with respect to $a$ is the unique linear map

$$
\partial_{a}: k Q \rightarrow k Q
$$

which sends a path $p$ to the sum $\sum_{p=u a v} v u$ taken over all decompositions of the path $p$ (where $u$ and $v$ are possibly idempotent elements $e_{i}$ associated to a vertex $i$ ). A potential on $Q$ is any (possibly infinite) linear combination $W$ of cycles in $Q$. The associated Jacobian algebra is

$$
\operatorname{Jac}(Q, W):=k \hat{Q} /\left\langle\partial_{a} W \mid a \in Q_{1}\right\rangle,
$$

where $k \hat{Q}$ is the completed path algebra (i.e., the completion of the path algebra $k Q$ at the ideal generated by the arrows of $Q$ ), and $\left\langle\partial_{a} W \mid a \in Q_{1}\right\rangle$ is the closure of the ideal generated by $\partial_{a} W$ for $a \in Q_{1}$.

A cluster category $\mathcal{C}(Q, W)$ associated with any quiver with potential $(Q, W)$ is constructed in [A, Section 3]. This construction uses the notion of Ginzburg dg algebras. We refer the reader to [A] for explicit details. When the associated Jacobian algebra is finite-dimensional, the category $\mathcal{C}(Q, W)$ is 2-Calabi-Yau and endowed with a canonical cluster-tilting object $T_{(Q, W)}$ whose endomorphism algebra is isomorphic to $\operatorname{Jac}(Q, W)$. The next result gives a link between cluster categories associated with algebra of global dimension at most 2 and cluster categories associated with quiver with potential.

TheOREM 2.5 ([K3, Theorem $6.12 \mathrm{a}]$ ). Let $\Lambda=k Q / I$ be a $\tau_{2}$-finite algebra of global dimension at most 2 such that $I$ is generated by a finite minimal set of relations $\left\{r_{i}\right\}$. (By this we mean that the set $\left\{r_{i}\right\}$ is the disjoint union of sets representing a basis of the $\mathrm{Ext}_{\Lambda}^{2}$-space between any two simple $\Lambda$ modules.) The relation $r_{i}$ starts at the vertex $s\left(r_{i}\right)$ and ends at the vertex $t\left(r_{i}\right)$. Then there is a triangle equivalence

$$
\mathcal{C}_{\Lambda} \cong \mathcal{C}(\bar{Q}, W)
$$

where the quiver $\bar{Q}$ is the quiver $Q$ with additional arrows $a_{i}: t\left(r_{i}\right) \rightarrow s\left(r_{i}\right)$, and the potential $W$ is $\sum_{i} a_{i} r_{i}$. This equivalence sends the cluster-tilting object $\pi(\Lambda)$ on the cluster-tilting object $T_{(\bar{Q}, W)}$.

As a consequence, we have an isomorphism of algebras:

$$
\operatorname{End}_{\mathcal{C}}(\pi \Lambda) \cong \operatorname{Jac}(\bar{Q}, W)
$$


Definition 2.6. A potential $W$ on a quiver $Q$ is said to be rigid if any cycle $p$ of $Q$ is cyclically equivalent to an element in the Jacobian ideal $\left\langle\partial_{a} W \mid a \in Q_{1}\right\rangle$.

By [DWZ, Corollary 6.11], rigidity is stable under mutation, and it implies that the Gabriel quiver of the Jacobian algebra has no loops or 2-cycles.

We end this section with two results linking the mutation of quivers with potential and mutation of cluster-tilting objects in cluster categories.

The first result links the mutation of cluster-tilting objects in the acyclic cluster category and the mutation of rigid quivers with potential defined in [DWZ, Section 5]. Acyclic cluster categories are equivalent to stable categories of some Frobenius categories associated to a certain reduced expression in the corresponding Coxeter group [BIRS1, Theorem II.3.4]. By [BIRS2, Corollary 6.7], these categories are all liftable (see [BIRS2, Section 5] for definition). Therefore, we get the following.

TheOREM 2.7 ([BIRS2, Corollary 5.4(b)]). Let $\Delta$ be an acyclic quiver, and let $T$ be a basic cluster-tilting object in the cluster category $\mathcal{C}_{\Delta}$. Assume that there exists a quiver with rigid potential $(Q, W)$ with an isomorphism

$$
\operatorname{End}_{\mathcal{C}_{\Delta}}(T) \cong \operatorname{Jac}(Q, W) .
$$

Let $i$ be a vertex of $Q$, and denote by $T_{i}$ the indecomposable summand of $T \cong T_{i} \oplus T^{\prime}$ corresponding to $i$. Then there is an isomorphism

$$
\operatorname{End}_{\mathcal{C}_{\Delta}}\left(\mu_{T_{i}}(T)\right) \cong \operatorname{Jac}\left(\mu_{i}(Q, W)\right)
$$

where $\mu_{T_{i}}(T)$ is defined in Theorem 2.2 and where $\mu_{i}(Q, W)$ is the mutation at $i$ of $(Q, W)$ as defined in [DWZ, Section 5] (see also Section 3.2 for definition).

The second result gives, for two quivers with potential linked by a mutation, an equivalence between the associated cluster categories.

TheOREM 2.8 ([KY, Theorem 3.2]). Let $(Q, W)$ be a quiver with rigid potential whose Jacobian algebra is finite-dimensional, and let $i \in Q_{0}$ be a vertex. Then there exists a triangle equivalence

$$
\mathcal{C}\left(\mu_{i}(Q, W)\right) \cong \mathcal{C}(Q, W)
$$

sending the cluster-tilting object $T_{\mu_{i}(Q, W)} \in \mathcal{C}\left(\mu_{i}(Q, W)\right)$ onto the clustertilting object $\mu_{T_{i}}\left(T_{(Q, W)}\right) \in \mathcal{C}(Q, W)$, where $T_{i}$ is the indecomposable summand of $T_{(Q, W)}$ associated with the vertex $i$ of $Q$. 
As a consequence, we get an isomorphism of algebras

$$
\operatorname{End}_{\mathcal{C}(Q, W)}\left(\mu_{T_{i}}\left(T_{(Q, W)}\right)\right) \cong \operatorname{Jac}\left(\mu_{i}(Q, W)\right)
$$

For a sequence $s=\left(i_{1}, \ldots, i_{r}\right)$ of vertices of $Q$, we denote by $\mu_{s}$ the composition $\mu_{i_{r}} \circ \mu_{i_{r-1}} \circ \cdots \circ \mu_{i_{1}}$, and we denote by $\mu_{s^{-}}$the composition $\mu_{i_{1}} \circ \mu_{i_{2}} \circ \cdots \circ \mu_{i_{r}}$.

For a bijection between $\{1, \ldots, n\}$ and the indecomposable summands of a basic cluster-tilting object $T$, we obtain a natural bijection between $\{1, \ldots, n\}$ and the indecomposable summands of $\mu_{i}(T)$. Therefore, once such a bijection is fixed, we will also use the notations $\mu_{s}$ and $\mu_{s^{-}}$for mutating cluster-tilting objects.

\subsection{Classical results on acyclic cluster categories}

In this article, we are interested in algebras of global dimension at most 2 whose generalized cluster category is equivalent to the cluster category associated to an acyclic quiver. Since we will make extensive use of them, we now recall some results for acyclic cluster categories.

The following theorem follows from a result by Happel and Unger [HU].

Theorem 2.9 ([BMRRT, Proposition 3.5]; see also [Hub]). Let $Q$ be an acyclic quiver, and let $T$ be a cluster-tilting object of $\mathcal{C}_{Q}$. Then there exists a sequence of mutations linking the cluster-tilting object $T$ to the canonical cluster-tilting object $\pi_{Q}(k Q)$. In other words, the cluster-tilting graph of the acyclic cluster category is connected.

A consequence of this theorem together with Theorem 2.7 is that the endomorphism algebra of a cluster-tilting object in an acyclic cluster category is always a Jacobian algebra of a rigid quiver with potential.

Another special feature of acyclic cluster categories is that they can be recognized by the quivers of their cluster-tilting objects.

THEOREM 2.10 ([KR, Section 2.1]). Let $\mathcal{C}$ be an algebraic triangulated category, which is Hom-finite and 2-Calabi-Yau. If there exists a clustertilting object $T \in \mathcal{C}$ such that $\operatorname{End}_{\mathcal{C}}(T) \cong k Q$, where $Q$ is an acyclic quiver, then there exists a triangle equivalence $\mathcal{C} \cong \mathcal{C}_{Q}$ sending $T$ onto $\pi_{Q}(k Q)$.

As a consequence, there is a triangle equivalence $\mathcal{C}(Q, 0) \rightarrow \mathcal{C}_{Q}$ sending $T_{(Q, 0)}$ onto $\pi_{Q}(k Q)$.

Note that an analogue of these results is not known for generalized cluster categories. 
An algebra of the form $\operatorname{End}_{\mathcal{C}_{Q}}\left(T^{\prime}\right)$, where $T^{\prime}$ is a cluster-tilting object in $\mathcal{C}_{Q}$, is called a cluster-tilted algebra of type $Q$.

\section{$\S 3$. Derived equivalent algebras of tree cluster type}

In this section, we investigate algebras of global dimension at most 2 whose generalized cluster category is a cluster category associated to a tree. We make extensive use of results from [AO1].

We start with a definition.

Definition 3.1 ([AO1, Definition 5.1]). Two algebras $\Lambda_{1}$ and $\Lambda_{2}$ of global dimension at most 2 which are $\tau_{2}$-finite are said to be cluster equivalent if there exists a triangle equivalence $\mathcal{C}_{\Lambda_{1}} \rightarrow \mathcal{C}_{\Lambda_{2}}$ between their generalized cluster categories.

If $\Lambda$ is cluster equivalent to $k Q$, where $Q$ is an acyclic quiver, we will say that $\Lambda$ is of cluster type $Q$.

Two derived equivalent algebras of global dimension at most 2 are cluster equivalent ([AO1, Corollary 7.16]). Hence, if the underlying graph of $Q$ is a tree, then the class of algebras of cluster type $Q$ does not depend on the orientation of $Q$.

From the results of Section 2, we deduce the following characterization of algebras of acyclic cluster type.

Corollary 3.2. Let $\Lambda=k Q / I$ be a $\tau_{2}$-finite algebra of global dimension at most 2. Let $(\bar{Q}, W)$ be the associated quiver with potential defined in Theorem 2.5. Then $\Lambda$ is of acyclic cluster type $\Delta$ if and only if there exists a sequence of mutation $s=i_{1}, \ldots, i_{l}$ such that $(\Delta, 0)=\mu_{s}(\bar{Q}, W)$.

In this case, $(\bar{Q}, W)$ is a rigid quiver with potential, and there exists a triangle equivalence $f: \mathcal{C}_{\Lambda} \rightarrow \mathcal{C}_{\Delta}$ sending $\pi_{\Lambda}(\Lambda)$ to $\mu_{s^{-}}\left(\pi_{\Delta}(k \Delta)\right)$.

Proof. By Theorems 2.5, 2.8, and 2.10, if $(\Delta, 0)=\mu_{s}(\bar{Q}, W)$, then we have equivalences

$$
\mathcal{C}_{\Lambda} \cong \mathcal{C}(\bar{Q}, W) \cong \mathcal{C}(\Delta, 0) \cong \mathcal{C}_{\Delta}
$$

sending $\pi_{\Lambda}(\Lambda)$ to $\mu_{s^{-}}\left(\pi_{\Delta}(k \Delta)\right)$.

Conversely, assume that there exists an equivalence $f: \mathcal{C}_{\Lambda} \cong \mathcal{C}_{\Delta}$. Then by Theorem 2.9 there exists a sequence of mutations $s$ such that $\pi_{\Delta}(k \Delta) \cong$ $\mu_{s} f\left(\pi_{\Lambda}(\Lambda)\right)$. So by Theorem 2.7, we have $\operatorname{Jac}\left(\mu_{s}(\bar{Q}, W)\right) \cong \operatorname{Jac}(\Delta, 0)=k \Delta$. Since the quiver with potential $\mu_{s}(\bar{Q}, W)$ is reduced, we necessarily have $\mu_{s}(\bar{Q}, W)=(\Delta, 0)$. 


\subsection{Graded equivalence and derived equivalence}

Cluster equivalence is strongly related to graded equivalence. In this section, we will recall some results shown in [AO1].

Let $A=\bigoplus_{p \in \mathbb{Z}} A^{p}$ be a finite-dimensional $\mathbb{Z}$-graded algebra. We denote by $d$ the degree map sending any homogeneous element of $A$ to its degree, and we denote by $\operatorname{gr} A$ the category of finitely generated graded modules over $A$. For a graded module $M=\bigoplus_{p \in \mathbb{Z}} M^{p}$, we denote by $M\langle q\rangle$ the graded module $\bigoplus_{p \in \mathbb{Z}} M^{p+q}$. (That is, the degree $p$ part of $M\langle q\rangle$ is $M^{p+q}$.) The locally bounded subcategory

$$
\operatorname{Cov}(A, d):=\operatorname{add}\{A\langle p\rangle \mid p \in \mathbb{Z}\} \subseteq \operatorname{gr} A
$$

is called the $\mathbb{Z}$-covering of $A$.

Definition 3.3. Let $A_{1}$ and $A_{2}$ be two finite-dimensional $\mathbb{Z}$-graded algebras. Assume that $A_{1}$ and $A_{2}$ are isomorphic as algebras. We will say that $A_{1}$ and $A_{2}$ are graded equivalent (and write $A_{1} \underset{\mathrm{gr}}{\sim} A_{2}$ ) if there exist $r_{i} \in \mathbb{Z}$ and an isomorphism of $\mathbb{Z}$-graded algebras

$$
A_{2} \underset{\mathbb{Z}}{\sim} \underset{p \in \mathbb{Z}}{\sim} \operatorname{Hom}_{\mathrm{Cov}\left(A_{1}, \mathbb{Z}\right)}\left(\bigoplus_{i=1}^{n} P_{i}\left\langle r_{i}\right\rangle, \bigoplus_{i=1}^{n} P_{i}\left\langle r_{i}+p\right\rangle\right),
$$

where $A_{1}=\bigoplus_{i=1}^{n} P_{i}$ is a decomposition of $A_{1}$ into indecomposable graded projective modules. This is equivalent to the fact that the coverings $\operatorname{Cov}\left(A_{1}\right.$, $\left.d_{1}\right)$ and $\operatorname{Cov}\left(A_{2}, d_{2}\right)$ are equivalent as categories with $\mathbb{Z}$-action.

The link between cluster equivalent algebras and graded equivalent algebras is given by the following result.

Theorem 3.4 ([AO1, Theorem 5.8]). Let $\Lambda_{1}$ and $\Lambda_{2}$ be two $\tau_{2}$-finite algebras of global dimension at most 2 . For $i=1,2$, denote by $\mathcal{D}_{i}$ the bounded derived category of $\Lambda_{i}$, denote by $\mathcal{C}_{i}$ its cluster category, and denote by $\pi_{i}$ the natural functor $\mathcal{D}_{i} \rightarrow \mathcal{C}_{i}$. Assume that there is $T \in \mathcal{D}_{1}$ such that $\pi_{1}(T)$ is basic cluster tilting in $\mathcal{C}_{1}$, and assume that

(1) there is an isomorphism $\operatorname{End}_{\mathcal{C}_{1}}\left(\pi_{1} T\right) \stackrel{\sim}{\longrightarrow} \operatorname{End}_{\mathcal{C}_{2}}\left(\pi_{2} \Lambda_{2}\right)$;

(2) this isomorphism can be chosen in such a way that the two $\mathbb{Z}$-gradings defined on $\bar{\Lambda}_{2}$, given respectively by

$$
\bigoplus_{q \in \mathbb{Z}} \operatorname{Hom}_{\mathcal{D}_{2}}\left(\Lambda_{2}, \mathbb{S}_{2}^{-q} \Lambda_{2}\right) \quad \text { and } \quad \bigoplus_{p \in \mathbb{Z}} \operatorname{Hom}_{\mathcal{D}_{1}}\left(T, \mathbb{S}_{2}^{-p} T\right)
$$


are equivalent. Then the algebras $\Lambda_{1}$ and $\Lambda_{2}$ are derived equivalent, and hence cluster equivalent.

Note that the functor $\mathbb{S}_{2}^{-1}$ acts on the subcategory $\pi_{1}^{-1} \pi_{1}(T)=\operatorname{add}\left\{\mathbb{S}_{2}^{-q} T\right.$, $q \in \mathbb{Z}\} \subset \mathcal{D}^{b}\left(\Lambda_{1}\right)$. As a category with $\mathbb{Z}$-action, it is equivalent to the $\mathbb{Z}$ covering of the graded algebra End $_{\mathcal{C}_{1}}\left(\pi_{1} T\right)$. Therefore, by the above remark, the graded algebras $\operatorname{End}_{\mathcal{C}_{1}}\left(\pi_{1} T\right)$ and $\operatorname{End}_{\mathcal{C}_{2}}\left(\pi_{2} \Lambda_{2}\right)$ are graded equivalent if and only if there is an equivalence between the additive categories $\pi_{1}^{-1} \pi_{1}(T)$ and $\pi_{2}^{-1} \pi_{2}\left(\Lambda_{2}\right)$ as categories with $\mathbb{S}_{2}$-action.

\subsection{Mutation of a graded quiver with potential}

In order to apply Theorem 3.4, we make use of a tool: the left (or right) mutation of a graded quiver with potential which extends the DerksenWeyman-Zelevinsky mutation of a quiver with potential (see [DWZ]). All definitions and results described in this section are proved in [AO1, Section 6].

Definition 3.5 ([AO1, Definition 6.4]). Let $(Q, W, d)$ be a $\mathbb{Z}$-graded quiver with potential homogeneous of degree 1 . Let $i \in Q_{0}$ be a vertex, such that there are neither loops nor 2-cycles incident to $i$. We define $\mu_{i}^{\mathrm{L}}(Q, W, d)$, the left graded mutation of $(Q, W, d)$ at vertex $i$, as the reduction of the graded QP $\left(Q^{\prime}, W^{\prime}, d^{\prime}\right)$. The quiver $Q^{\prime}$ is defined as in [DWZ, Section 5] as follows.

- For any subquiver $u \stackrel{a}{\longrightarrow} i \stackrel{b}{\longrightarrow} v$, with $i, u$, and $v$ pairwise distinct vertices, we add an arrow $[b a]: u \rightarrow v$.

- We replace all arrows $a$ incident with $i$ by an arrow $a^{*}$ in the opposite direction.

The potential $W^{\prime}$ is also defined as in [DWZ, Section 5] by the sum $[W]+$ $W^{*}$, where $[W]$ is formed from the potential $W$ replacing all compositions $b a$ through the vertex $i$ by $[b a]$, where $W^{*}$ is the sum $\sum a^{*} b^{*}[b a]$.

The new degree $d^{\prime}$ is defined as

- $d^{\prime}(a)=d(a)$ if $a$ is an arrow of $Q$ and $Q^{\prime}$;

- $d^{\prime}([b a])=d(b)+d(a)$ if $b a$ is a composition passing through $i$;

- $d^{\prime}\left(a^{*}\right)=-d(a)+1$ if the target of $a$ is $i$;

- $d^{\prime}\left(b^{*}\right)=-d(b)$ if the source of $b$ is $i$.

One can check that this operation is compatible with the reduction of a QP (see [AO1, Theorem 6.6]). It is possible to define the right graded mutation $\mu_{i}^{\mathrm{R}}$ by interchanging target and source in the last two items in the definition. We have the following. 
LEMMA 3.6 ([AO1, Lemma 6.9]). Let $(Q, W, d)$ be a graded quiver with potential. Then we have an isomorphism of $\mathbb{Z}$-graded algebras

$$
\operatorname{Jac}(Q, W, d) \cong \underset{\mathbb{Z}}{ } \operatorname{Jac}\left(\mu_{i}^{\mathrm{R}} \mu_{i}^{\mathrm{L}}(Q, W, d)\right)
$$

Moreover, this mutation preserves graded equivalence. More precisely, we deduce immediately the following from the definition of graded quiver with potential.

Proposition 3.7. Let $\left(Q, W, d_{1}\right)$ and $\left(Q, W, d_{2}\right)$ be two $\mathbb{Z}$-graded $Q P s$ such that the graded Jacobian algebras $\operatorname{Jac}\left(Q, W, d_{1}\right)$ and $\operatorname{Jac}\left(Q, W, d_{2}\right)$ are graded equivalent. Then the graded Jacobian algebras $\operatorname{Jac}\left(\mu_{i}^{\mathrm{L}}\left(Q, W, d_{1}\right)\right)$ and $\operatorname{Jac}\left(\mu_{i}^{\mathrm{L}}\left(Q, W, d_{2}\right)\right)$ are graded equivalent.

Definition 3.8 ([AO1, Theorem 2.6]). Let $\Lambda$ be a $\tau_{2}$-finite algebra of global dimension at most 2. Let $T=T_{1} \oplus \cdots \oplus T_{n}$ be an object in $\mathcal{D}^{b}(\Lambda)$ such that $\pi(T)$ is a (basic) cluster-tilting object in $\mathcal{C}_{\Lambda}$. Let $T_{i}$ be an indecomposable summand of $T \cong T^{\prime} \oplus T_{i}$. Define $T_{i}^{\mathrm{L}}$ as the cone in $\mathcal{D}^{b}(\Lambda)$ of the minimal left add $\left\{\mathbb{S}_{2}^{p} T^{\prime}, p \in \mathbb{Z}\right\}$-approximation $u: T_{i} \rightarrow B$ of $T_{i}$. We denote by $\mu_{i}^{\mathrm{L}}(T)$ the object $T_{i}^{\mathrm{L}} \oplus T^{\prime}$ and call it the left mutation of $T$ at $T_{i}$.

It is immediate to check that we have $\pi\left(\mu_{i}^{\mathrm{L}}(T)\right)=\mu_{i}(\pi(T))$; thus, $\pi\left(\mu_{i}^{\mathrm{L}}(T)\right)$ is a cluster-tilting object in $\mathcal{C}_{\Lambda}$.

This (left) mutation in the derived category is reflected by the graded (left) mutation of graded QP in the following sense.

THEOREM 3.9. Let $\Lambda$ be an algebra of acyclic cluster type, and let $T \in$ $\mathcal{D}^{b}(\Lambda)$ be as described above. Assume that there exists a $\mathbb{Z}$-graded $Q P(Q$, $W, d)$ with rigid potential homogeneous of degree 1 such that we have an isomorphism of graded algebras

$$
\bigoplus_{p \in \mathbb{Z}} \operatorname{Hom}_{\mathcal{D}}\left(T, \mathbb{S}_{2}^{-p} T\right) \underset{\mathbb{Z}}{\stackrel{\sim}{\longrightarrow}} \operatorname{Jac}(Q, W, d)
$$

Let $i \in Q_{0}$, and let $T_{i}$ be the associated indecomposable summand of $T \cong$ $T_{i} \oplus T^{\prime}$. Then there is an isomorphism of $\mathbb{Z}$-graded algebras

$$
\bigoplus_{p \in \mathbb{Z}} \operatorname{Hom}_{\mathcal{D}}\left(T^{\prime} \oplus T_{i}^{\mathrm{L}}, \mathbb{S}_{2}^{-p}\left(T^{\prime} \oplus T_{i}^{\mathrm{L}}\right)\right) \underset{\mathbb{Z}}{\stackrel{\sim}{\longrightarrow}} \operatorname{Jac}\left(\mu_{i}^{\mathrm{L}}(Q, W, d)\right)
$$


Proof. By Theorem 2.7, we already have an isomorphism between the algebras

$$
\bigoplus_{p \in \mathbb{Z}} \operatorname{Hom}_{\mathcal{D}}\left(T^{\prime} \oplus T_{i}^{\mathrm{L}}, \mathbb{S}_{2}^{-p}\left(T^{\prime} \oplus T_{i}^{\mathrm{L}}\right)\right) \cong \operatorname{End}_{\mathcal{C}_{\Lambda}}\left(\mu_{\pi\left(T_{i}\right)}(\pi T)\right)
$$

and $\operatorname{Jac}\left(\mu_{i}^{\mathrm{L}}(Q, W)\right)$ forgetting the grading. The only thing to check is that it is compatible with the grading. The proof of this fact is the same as the one of [AO1, Theorem 6.12].

Definition 3.10. Let $(Q, W)$ be a reduced quiver with potential. A grading $d$ on $Q$ will be said to be a $W$-grading if

- for all arrows $a$ in $Q_{1}, d(a) \in\{0,1\}$;

- the potential $W$ is homogeneous of degree 1 ;

- the set of relations $\left\{\partial_{a} W \mid d(a)=1\right\}$ is linearly independent (in particular, for all $a \in Q_{1}$ such that $d(a)=1$, we have $\left.\partial_{a} W \neq 0\right)$.

Theorem 3.9 is particularly useful with the following result.

Proposition 3.11 ([AO1, Proposition 6.3]). Let $\Lambda=k Q_{\Lambda} / I$ be an algebra of global dimension at most 2 which is $\tau_{2}$-finite and of acyclic cluster type. Then there exists a rigid quiver with potential $(\bar{Q}, W)$ and a $W$-grading $d$ such that we have an isomorphism of graded algebras

$$
\bar{\Lambda} \underset{\mathbb{Z}}{\cong} \operatorname{Jac}(\bar{Q}, W, d)
$$

The quiver with potential is given by Theorem 2.5; the arrows of $Q_{\Lambda}$ have degree 0 , and the arrows $a_{i}$ corresponding to the relations have degree 1 . The rigidity comes from Corollary 3.2.

\subsection{Application to acyclic cluster type}

Let $\Lambda$ be an algebra of cluster type $Q$, where $Q$ is an acyclic quiver. By Corollary 3.2, there exists a sequence of mutations $s$ such that $\mu_{s}\left(Q_{\bar{\Lambda}}, W\right)=$ $(Q, 0)$, where $\left(Q_{\bar{\Lambda}}, W\right)$ is the quiver with potential associated with $\Lambda$ in Theorem 2.5.

Definition 3.12. The map $d_{s}: Q \rightarrow \mathbb{Z}$ is called a grading induced by $\Lambda$ if it satisfies

$$
\mu_{s}^{\mathrm{L}}\left(Q_{\bar{\Lambda}}, W, d\right)=\left(Q, 0, d_{s}\right)
$$

where $s$ is a sequence of mutations as in Corollary 3.2. 
Proposition 3.13. Let $d_{s}$ and $d_{t}$ be two gradings induced by $\Lambda$ on $Q$. If $\mu_{s}\left(\pi_{\Lambda}(\Lambda)\right) \cong \mu_{t}\left(\pi_{\Lambda}(\Lambda)\right)$, then $d_{s}$ and $d_{t}$ are equivalent up to automorphism of $Q$.

Proof. Let $\mu_{s}^{\mathrm{L}} \Lambda \cong T_{1} \oplus \cdots \oplus T_{n}$ be a decomposition of $\mu_{s}^{\mathrm{L}} \Lambda$ into indecomposable summands. Then we have

$$
\mu_{t}^{\mathrm{L}} \Lambda \cong \mathbb{S}_{2}^{r_{1}} T_{1} \oplus \cdots \oplus \mathbb{S}_{2}^{r_{n}} T_{n}
$$

for certain $r_{i} \in \mathbb{Z}$, since $\pi_{\Lambda} \mu_{s}^{\mathrm{L}} \Lambda=\mu_{s} \pi_{\Lambda} \Lambda \cong \mu_{t} \pi_{\Lambda} \Lambda=\pi_{\Lambda} \mu_{t}^{\mathrm{L}} \Lambda$. It follows that the algebras

$$
\bigoplus_{p \in \mathbb{Z}} \operatorname{Hom}_{\mathcal{D}^{b}(\Lambda)}\left(\mu_{s}^{\mathrm{L}} \Lambda, \mathbb{S}_{2}^{-p} \mu_{s}^{\mathrm{L}} \Lambda\right) \quad \text { and } \quad \bigoplus_{p \in \mathbb{Z}} \operatorname{Hom}_{\mathcal{D}^{b}(\Lambda)}\left(\mu_{t}^{\mathrm{L}} \Lambda, \mathbb{S}_{2}^{-p} \mu_{t}^{\mathrm{L}} \Lambda\right)
$$

are graded equivalent. Since, by Theorem 3.9, these graded algebras are isomorphic to $\operatorname{Jac}\left(Q, 0, d_{s}\right)$ and $\operatorname{Jac}\left(Q, 0, d_{t}\right)$, respectively, it follows that the gradings $d_{s}$ and $d_{t}$ are equivalent up to automorphisms of $Q$.

REMARK 3.14. The induced grading depends in general on the choice of a cluster-tilting object in $\mathcal{C}_{\Lambda}$ with hereditary endomorphism ring, as shown in the following example. Let $\Lambda$ be the algebra presented by the quiver

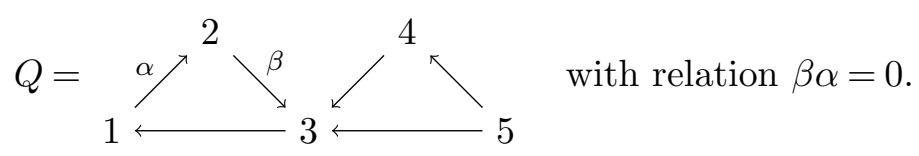

Adding an arrow of degree 1 for the relation, one obtains a graded quiver with potential $(\bar{Q}, W, d)$. Then it is easy to check that $\mu_{2}^{\mathrm{L}}(\bar{Q}, W, d)$ is given by the graded quiver

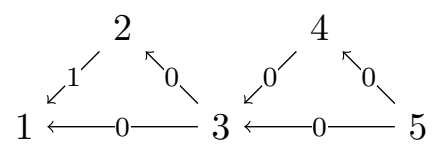

On the other hand, $\mu_{2}^{\mathrm{L}} \mu_{1}^{\mathrm{L}} \mu_{4}^{\mathrm{L}} \mu_{5}^{\mathrm{L}} \mu_{2}^{\mathrm{L}}(\bar{Q}, W, d)$ is given by the graded quiver

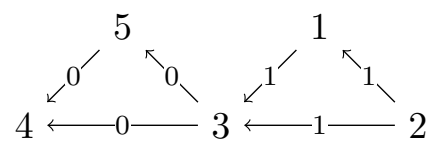

These gradings are not equivalent. 
Using the previous results, we can prove the following.

THEOREM 3.15. Let $\Lambda_{1}$ and $\Lambda_{2}$ be two algebras of cluster type $Q$, where $Q$ is an acyclic quiver. Then the following are equivalent.

(1) There exist equivalent gradings $d_{s_{i}}$ induced by $\Lambda_{i}$ on $Q$ for $i \in\{1,2\}$.

(2) There exists a derived equivalence $\mathcal{D}^{b}\left(\Lambda_{1}\right) \rightarrow \mathcal{D}^{b}\left(\Lambda_{2}\right)$.

Proof. For $i=1,2$, denote by $\left(Q_{i}, W_{i}, \partial_{i}\right)$ the graded QP associated to $\bar{\Lambda}_{i}$. $(1) \Rightarrow(2)$ By assumption, we have a graded equivalence

$$
\operatorname{Jac}\left(\mu_{s_{1}}^{\mathrm{L}}\left(Q_{1}, W_{1}, \partial_{1}\right)\right) \underset{\mathrm{gr}}{\sim} \operatorname{Jac}\left(\mu_{s_{2}}^{\mathrm{L}}\left(Q_{2}, W_{2}, \partial_{2}\right)\right) .
$$

Then by Proposition 3.7 and Lemma 3.6, we immediately get that

$$
\operatorname{Jac}\left(\mu_{s_{2}^{-}}^{\mathrm{R}} \mu_{s_{1}}^{\mathrm{L}}\left(Q_{1}, W_{1}, \partial_{1}\right)\right) \underset{\mathrm{gr}}{\sim} \operatorname{Jac}\left(Q_{2}, W_{2}, \partial_{2}\right)
$$

Now denote by $T \in \mathcal{D}^{b}\left(\Lambda_{1}\right)$ the object $\mu_{s_{2}^{-}}^{\mathrm{R}} \mu_{s_{1}}^{\mathrm{L}}\left(\Lambda_{1}\right)$. By Theorem 3.9 we have an isomorphism of $\mathbb{Z}$-graded algebras

$$
\bigoplus_{p \in \mathbb{Z}} \operatorname{Hom}_{\mathcal{D}^{b}\left(\Lambda_{1}\right)}\left(T, \mathbb{S}_{2}^{-p} T\right) \underset{\mathbb{Z}}{\cong} \operatorname{Jac}\left(\mu_{s_{2}^{-}}^{\mathrm{R}} \mu_{s_{1}}^{\mathrm{L}}\left(Q_{1}, W_{1}, \partial_{1}\right)\right) .
$$

Therefore, we get the result by Theorem 3.4.

$(2) \Rightarrow(1)$ Assume that $\Lambda_{1}$ and $\Lambda_{2}$ are derived equivalent. Then there exists a tilting complex $T$ in $\mathcal{D}^{b}\left(\Lambda_{2}\right)$ with $\operatorname{End}_{\mathcal{D}^{b}\left(\Lambda_{2}\right)}(T) \cong \Lambda_{1}$. The derived equivalence induced by $T$ gives rise to a commutative diagram

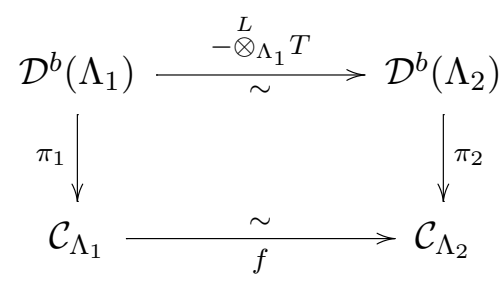

Since the cluster category $\mathcal{C}_{\Lambda_{2}}$ is acyclic, there exists a sequence of mutations $s$ such that $\mu_{s}\left(\pi_{2} \Lambda_{2}\right)=\pi_{2}(T)=f\left(\pi_{1} \Lambda_{1}\right)$. Denote by $T^{\prime} \in \mathcal{D}^{b}\left(\Lambda_{2}\right)$ the object $T^{\prime}:=\mu_{s}^{\mathrm{L}}\left(\Lambda_{2}\right)$. Then we have $\pi_{2}(T)=\pi_{2}\left(T^{\prime}\right)$; thus, we have an equivalence of categories commuting with $\mathbb{S}_{2}$

$$
\operatorname{add}\left\{\mathbb{S}_{2}^{p} T \mid p \in \mathbb{Z}\right\} \cong \operatorname{add}\left\{\mathbb{S}_{2}^{p} T^{\prime} \mid p \in \mathbb{Z}\right\}
$$


This exactly means that there is a graded equivalence

$$
\bigoplus_{p \in \mathbb{Z}} \operatorname{Hom}_{\mathcal{D}^{b}\left(\Lambda_{2}\right)}\left(T, \mathbb{S}_{2}^{-p} T\right) \underset{\mathrm{gr}}{\sim} \bigoplus_{p \in \mathbb{Z}} \operatorname{Hom}_{\mathcal{D}^{b}\left(\Lambda_{2}\right)}\left(T^{\prime}, \mathbb{S}_{2}^{-p} T^{\prime}\right) .
$$

Since $-\stackrel{L}{\otimes}_{\Lambda_{1}} T$ is an equivalence, the left term is isomorphic as $\mathbb{Z}$-graded algebra to $\operatorname{Jac}\left(Q_{1}, W_{1}, \partial_{1}\right)$, and by Theorem 3.9 the right term is isomorphic to the $\mathbb{Z}$-graded algebra $\operatorname{Jac}\left(\mu_{s}^{\mathrm{L}}\left(Q_{2}, W_{2}, \partial_{2}\right)\right)$. Therefore, we have

$$
\operatorname{Jac}\left(\mu_{s}^{\mathrm{L}}\left(Q_{2}, W_{2}, \partial_{2}\right)\right) \underset{\mathrm{gr}}{\sim} \operatorname{Jac}\left(Q_{1}, W_{1}, \partial_{1}\right)
$$

Now let $s^{\prime}$ be a sequence such that the cluster-tilting object $T^{\prime \prime}:=\mu_{s^{\prime}}\left(f \pi_{1} \Lambda_{1}\right)$ has endomorphism algebra isomorphic to $k Q$. Then we have

$$
\mu_{s^{\prime}}\left(Q_{1}, W_{1}\right)=(Q, 0) \quad \text { and } \quad \mu_{s^{\prime}} \mu_{s}\left(Q_{2}, W_{2}\right)=(Q, 0)
$$

Now let $d_{1}$ and $d_{2}$ be the gradings on $Q$ such that we have

$$
\mu_{s^{\prime}}^{\mathrm{L}}\left(Q_{1}, W_{1}, \partial_{1}\right)=\left(Q, 0, d_{1}\right) \quad \text { and } \quad \mu_{s^{\prime}}^{\mathrm{L}} \mu_{s}^{\mathrm{L}}\left(Q_{2}, W_{2}, \partial_{2}\right)=\left(Q, 0, d_{2}\right)
$$

By (3.3.1) and Proposition 3.7, we get

$$
\operatorname{Jac}\left(Q, 0, d_{1}\right) \underset{\mathrm{gr}}{\sim} \operatorname{Jac}\left(Q, 0, d_{2}\right)
$$

that is, the gradings $d_{1}$ and $d_{2}$ are equivalent.

Corollary 3.16. If $Q$ is a tree, and if $\Lambda$ is of cluster type $Q$, then $\Lambda$ is derived equivalent to $k Q$.

Proof. By Theorem 3.15, it is enough to observe that all gradings on $k Q$ are equivalent. We consider the map

$$
\begin{aligned}
\mathbb{Z}^{Q_{0}} & \rightarrow \mathbb{Z}^{Q_{1}} \\
\left(r_{i}\right)_{i \in Q_{0}} & \mapsto\left(r_{t(a)}-r_{s(a)}\right)_{a \in Q_{1}} .
\end{aligned}
$$

Then the grading on $Q$ which is equivalent to the trivial grading is the image of this map. Moreover, using the fact that $Q$ is a tree, one easily sees that the map is surjective. 


\section{§4. Derived equivalence classes of algebras of cluster type $\widetilde{A}_{p, q}$}

In this section we study the algebras of cluster type $\widetilde{A}_{p, q}$, that is, all algebras which are cluster equivalent to the path algebra $H$ of the acyclic quiver $Q_{H}$ :

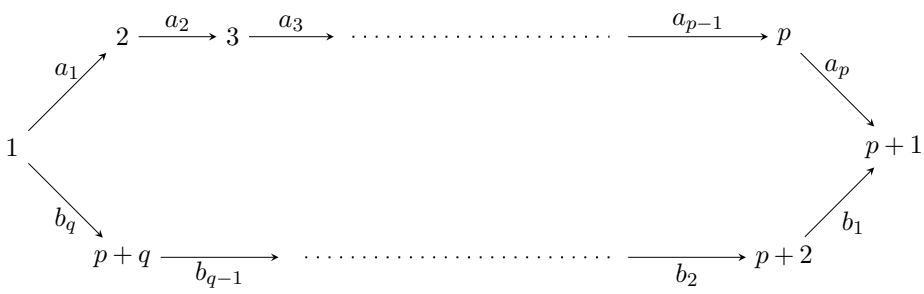

Since $Q_{H}$ is not a tree, these algebras need not be derived equivalent. In this section, we introduce an invariant of an algebra of cluster type $Q_{H}$ which determines its class of derived equivalence.

\subsection{The weight of an algebra of cluster type $\widetilde{A}_{p, q}$}

Definition 4.1. Let $d$ be a $\mathbb{Z}$-grading on $Q_{H}$. We define the weight of the grading $d$ by

$$
w(H, d):=\sum_{i=1}^{p} d\left(a_{i}\right)-\sum_{i=1}^{q} d\left(b_{i}\right) .
$$

Lemma 4.2. Let $d_{1}$ and $d_{2}$ be two $\mathbb{Z}$-gradings on $H$. Then the following are equivalent:

(1) $\left(H, d_{1}\right)$ and $\left(H, d_{2}\right)$ are graded equivalent,

(2) $w\left(H, d_{1}\right)=w\left(H, d_{2}\right)$.

Proof. We consider the map

$$
\begin{aligned}
\phi: \mathbb{Z}^{\left(Q_{H}\right)_{0}} & \rightarrow \mathbb{Z}^{\left(Q_{H}\right)_{1}} \\
\left(r_{i}\right)_{i \in\left(Q_{H}\right)_{0}} & \mapsto\left(r_{t(a)}-r_{s(a)}\right)_{a \in\left(Q_{H}\right)_{1}}
\end{aligned}
$$

The gradings $d_{1}$ and $d_{2}$ are equivalent if and only if $d_{1}-d_{2}$ is in the image of $\phi$.

It is straightforward to check that the sequence of $\mathbb{Z}$-modules

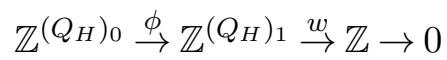

is exact (using the fact that $Q$ is of type $\widetilde{A}$ ). Now the claim of the lemma follows. 
This result allows us to define the following.

Definition 4.3. Let $\Lambda$ be an algebra of global dimension at most 2 and of cluster type $\widetilde{A}_{p, q}$. Let $s$ be a sequence of mutations such that the endomorphism ring of $\mu_{s}\left(\pi_{\Lambda}(\Lambda)\right)$ is $k Q_{H}$ (such a sequence exists by Corollary 3.2), and let $d_{s}$ be the corresponding grading on $Q_{H}$ induced by $\Lambda$. Then define the weight of $\Lambda$ by

$$
w(\Lambda, s):=w\left(d_{s}\right)=\sum_{i=1}^{p} d_{s}\left(a_{i}\right)-\sum_{i=1}^{q} d_{s}\left(b_{i}\right) .
$$

If $p>q$, the weight $w(\Lambda, s)$ is well defined since there is no automorphism of $Q_{H}$.

\subsection{The case $p>q$}

Before proving the main theorem, we prove the following technical result.

Lemma 4.4. The weight $w(\Lambda, s)$ does not depend on the choice of the sequence of mutations $s$.

Proof. Let $s$ and $t$ be two sequences of mutations such that the endomorphism rings of $\mu_{s}\left(\pi_{\Lambda}(\Lambda)\right)$ and of $\mu_{t}\left(\pi_{\Lambda}(\Lambda)\right)$ are both isomorphic to $k Q_{H}$. Then there is an automorphism of the translation quiver $\mathbb{Z} Q_{H}$, which forms a component of the Auslander-Reiten quiver of the common cluster category, mapping $\mu_{s}\left(\pi_{\Lambda}(\Lambda)\right)$ to $\mu_{t}\left(\pi_{\Lambda}(\Lambda)\right)$. Since $p \neq q$, one can check that the automorphism group of the translation quiver $\mathbb{Z} Q_{H}$ is generated by two elements given by the slices

$$
\begin{aligned}
T_{1}= & \tau^{-1}\left(e_{1} H \oplus \cdots \oplus e_{p} H\right) \oplus e_{p+q} H \oplus \cdots \oplus e_{p+1} H, \quad \text { and } \\
T_{2}= & e_{2} H \oplus \cdots \oplus e_{p+1} H \\
& \oplus \tau^{-1}\left(e_{1} H \oplus e_{p+q} H \oplus e_{p+q-1} H \oplus \cdots \oplus e_{p+2} H\right), \quad \text { respectively, }
\end{aligned}
$$

where $e_{i}$ is the primitive idempotent of $H=k Q$ associated to the vertex $i$ for any $i=1, \ldots, p+q$. Then we have

$$
T_{1}=\mu_{p}^{\mathrm{L}} \mu_{p-1}^{\mathrm{L}} \cdots \mu_{1}^{\mathrm{L}}(H) \quad \text { and } \quad T_{2}=\mu_{p+2}^{\mathrm{L}} \mu_{p+3}^{\mathrm{L}} \cdots \mu_{p+q}^{\mathrm{L}} \mu_{1}^{\mathrm{L}}(H) .
$$

It is enough to check that if $d$ is a grading on $Q_{H}$, then the weights of the graded quivers $\mu_{p}^{\mathrm{L}} \mu_{p-1}^{\mathrm{L}} \cdots \mu_{1}^{\mathrm{L}}\left(Q_{H}, d\right)$ and $\mu_{p+2}^{\mathrm{L}} \mu_{p+3}^{\mathrm{L}} \cdots \mu_{p+q}^{\mathrm{L}} \mu_{1}^{\mathrm{L}}\left(Q_{H}, d\right)$ are equal to $w(d)$. We do this for $\mu_{p}^{\mathrm{L}} \mu_{p-1}^{\mathrm{L}} \cdots \mu_{1}^{\mathrm{L}}\left(Q_{H}, d\right)$; the other one is similar. 
First note that in the sequence of mutations $\mu_{p}^{\mathrm{L}} \mu_{p-1}^{\mathrm{L}} \cdots \mu_{1}^{\mathrm{L}}$, we mutate at a source at each step. Therefore, the left graded mutation consists in reversing the arrows and assigning the opposite of the degree. After the sequence of mutations, arrows $a_{1}, \ldots, a_{p-1}$ have been reversed twice, arrows $a_{p}$ and $b_{q}$ have been reversed once, and $b_{1}, \ldots, b_{q}$ have not been reversed. Hence, the graded quiver $\mu_{p}^{\mathrm{L}} \mu_{p-1}^{\mathrm{L}} \cdots \mu_{1}^{\mathrm{L}}\left(Q_{H}, d\right)$ is the following:

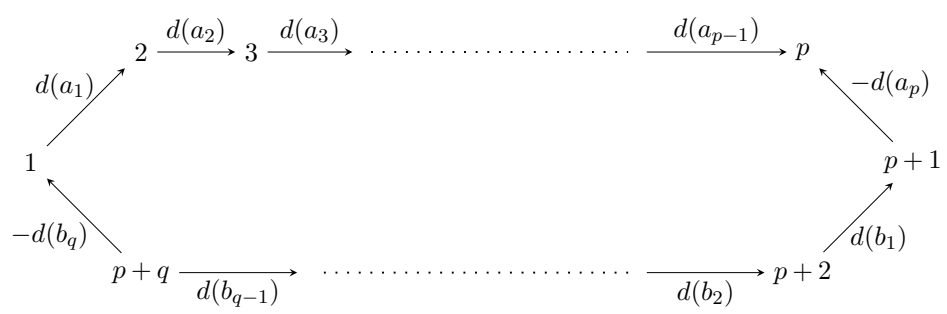

Hence, the weight of this grading is $\left(-d\left(b_{q}\right)+\sum_{i=1}^{p-1} d\left(a_{i}\right)\right)-\left(\sum_{j=1}^{q-1} d\left(b_{j}\right)-\right.$ $\left.d\left(a_{p}\right)\right)=w(d)$.

This lemma together with Lemma 4.2 shows that the situation in Remark 3.14 does not occur for a quiver of type $\widetilde{A}_{p, q}$. Hence, we may, in the sequel, refer to the weight $w(\Lambda)$ without specifying a sequence of mutations.

THEOREM 4.5. Let $\Lambda_{1}$ and $\Lambda_{2}$ be two algebras of cluster type $\widetilde{A}_{p, q}$ with $p>q$. Then there is a derived equivalence between $\Lambda_{1}$ and $\Lambda_{2}$ if and only if $w\left(\Lambda_{1}\right)=w\left(\Lambda_{2}\right)$.

Proof. Let $\delta_{1}$ (resp., $\delta_{2}$ ) be a grading on $Q_{H}$ induced by $\Lambda_{1}$ (resp., $\Lambda_{2}$ ). By Lemma $4.2, \delta_{1}$ and $\delta_{2}$ are equivalent if and only if the corresponding weights $w\left(\Lambda_{1}\right)$ and $w\left(\Lambda_{2}\right)$ coincide. Since, by Lemma 4.4 , the weights are independent of the choice of a sequence of mutation, it follows that also the gradings $\delta_{1}$ and $\delta_{2}$ are independent of this choice up to graded equivalence. Now the claim follows from Theorem 3.15.

Corollary 4.6. Let $\Lambda$ be an algebra of cluster type $\widetilde{A}_{p, q}$. Then $\Lambda$ is piecewise hereditary if and only if $w(\Lambda)=0$.

Proof. Let us treat the case where $p>q$. The "if" part of the assertion is a direct consequence of Theorem 4.5 applied for $\Lambda_{1}=\Lambda$ and $\Lambda_{2}=H$.

The algebra $\Lambda$ is piecewise hereditary if and only if the derived category $\mathcal{D}^{b}(\Lambda)$ is equivalent to $\mathcal{D}^{b}(\mathcal{H})$ for some hereditary category $\mathcal{H}$. Therefore, it implies that the generalized cluster category $\mathcal{C}_{\Lambda}$ is equivalent to the cluster 
category $\mathcal{C}_{\mathcal{H}}$. Since $\Lambda$ is of cluster type $\widetilde{A}_{p, q}$, we have $\mathcal{C}_{\mathcal{H}} \cong \mathcal{C}_{\widetilde{A}_{p, q}}$. Hence, we get $\mathcal{D}^{b}(\mathcal{H}) \cong \mathcal{D}^{b}\left(\widetilde{A}_{p, q}\right)$. Therefore, by Theorem 4.5 , we have $w(\Lambda)=0$.

The case $p=q$ is a direct consequence of the result below.

\subsection{The case $p=q$}

In the case $p=q$, there is a unique nontrivial automorphism of $Q_{H}$ fixing the vertices 1 and $p+1$ and interchanging the vertices $i$ and $2 p+2-i$ for $i=$ $2, \ldots, p$. This automorphism induces a derived equivalence between algebras $\Lambda_{1}$ and $\Lambda_{2}$ of cluster type $\widetilde{A}_{p, p}$ such that $w\left(\Lambda_{1}\right)=-w\left(\Lambda_{2}\right)$. Therefore, we obtain the following result, whose proof is the same as that of Theorem 4.5.

THEOREM 4.7. Let $\Lambda_{1}$ and $\Lambda_{2}$ be two algebras of cluster type $\widetilde{A}_{p, p}$. Then there is a derived equivalence between $\Lambda_{1}$ and $\Lambda_{2}$ if and only if $\left|w\left(\Lambda_{1}\right)\right|=$ $\left|w\left(\Lambda_{2}\right)\right|$.

\section{§5. The Auslander-Reiten quiver of the derived category of an algebra of cluster type $\widetilde{A}_{p, q}$}

Let $\Lambda$ be an algebra of cluster type $\widetilde{A}_{p, q}$ and of weight $w \neq 0$. In this section, we compute the Auslander-Reiten quiver of the derived category $\mathcal{D}^{b}(\Lambda)$. In order to do that, we use some results of [AO1, Section 8].

\subsection{Graded derived equivalence}

Let $\Lambda$ be an algebra of cluster type $\widetilde{A}_{p, q}$. Then, by Proposition 3.11 , there exists a graded quiver with reduced potential $(\bar{Q}, W, d)$ such that we have an isomorphism of $\mathbb{Z}$-graded algebras $\bar{\Lambda} \cong \operatorname{Jac}(\bar{Q}, W, d)$. Let $\partial$ be a grading induced by $\Lambda$ on $H$ where $Q_{H}$ is the following quiver:

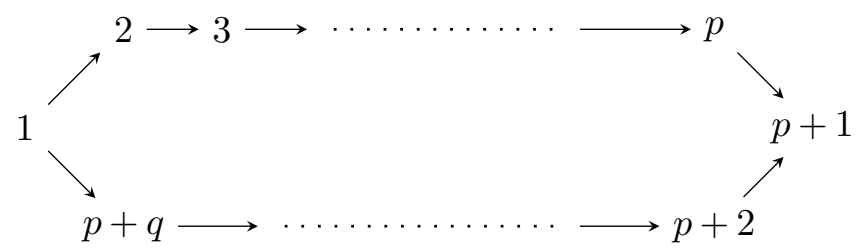

That is, there exists a sequence of mutations such that $\mu_{s}(\bar{Q}, W, d)=\left(Q_{H}\right.$, $0, \partial)$. Now define the $\mathbb{Z}^{2}$-graded quiver with reduced potential $\left(Q^{\prime}, W^{\prime}\right.$, $\left.\left(d^{\prime}, \delta\right)\right)$ by the following:

$$
\left(Q^{\prime}, W^{\prime},\left(d^{\prime}, \delta\right)\right):=\mu_{s^{-}}^{\mathrm{R}}\left(Q_{H}, 0,(\partial, 0)\right)
$$


By Lemma 3.6, we have an isomorphism of $\mathbb{Z}$-graded algebras $\operatorname{Jac}\left(Q^{\prime}, W^{\prime}\right.$, $\left.d^{\prime}\right) \underset{\mathbb{Z}}{\cong} \operatorname{Jac}(\bar{Q}, W, d)$. Since the potentials $W$ and $W^{\prime}$ are reduced, the quivers $\bar{Q}$ and $Q^{\prime}$ are isomorphic. By definition of $\bar{Q}$ and $d$, the quiver $Q_{\Lambda}$ of the algebra $\Lambda$ is the subquiver of $\bar{Q}$ satisfying $\left(Q_{\Lambda}\right)_{0}=\bar{Q}_{0}$ and $\left(Q_{\Lambda}\right)_{1}=\left\{a \in \bar{Q}_{1} \mid d(a)=\right.$ $0\}$. Moreover, we have an isomorphism $\Lambda \cong k Q_{\Lambda} /\left\langle\partial_{a} W, a \in \bar{Q}_{1}, d(a)=1\right\rangle$. Since we have an isomorphism of $\mathbb{Z}$-graded algebras $\operatorname{Jac}\left(Q^{\prime}, W^{\prime}, d^{\prime}\right) \cong \underset{\mathbb{Z}}{ } \operatorname{Jac}(\bar{Q}$, $W, d)$, we get an isomorphism $\Lambda \cong k Q_{\Lambda} /\left\langle\partial_{a} W^{\prime}, a \in Q_{1}^{\prime}, d^{\prime}(a)=1\right\rangle$. The grading $\delta$, which is a grading on $Q^{\prime} \cong \bar{Q}$, restricts on a grading on $Q_{\Lambda}$. The grading $\delta$ makes $W^{\prime}$ homogeneous of degree 1 . Hence, the relations $\partial_{a} W^{\prime}, a \in Q_{1}^{\prime}$ with $d^{\prime}(a)=1$ are homogeneous with respect to the degree $\delta$. Consequently, $\delta$ yields a grading on the algebra $\Lambda$ that we still denote by $\delta$.

Then we have the following direct consequence.

THEOREM 5.1 ([AO1, Theorem 8.7]). In the preceding setup, there exists a triangle equivalence

$$
\mathcal{D}^{b}(\operatorname{Cov}(\Lambda, \delta)) \stackrel{F}{\sim} \mathcal{D}^{b}(\operatorname{Cov}(H, \partial))
$$

Moreover, we have an isomorphism of triangle functors $F \circ\langle 1\rangle_{\delta} \cong \mathbb{S}_{2}^{-1} \circ$ $\langle-1\rangle_{\partial} \circ F$.

REMARK 5.2. Note that in this situation, the compatibility condition defined in [AO1, Definition 8.5] is automatically satisfied since $\bar{Q}$ is mutation acyclic and since $W$ is rigid. Indeed, two $\mathbb{Z}$-gradings on a quiver induce a $\mathbb{Z}^{2}$-grading on it. But in general, two $\mathbb{Z}$-gradings on an algebra do not give rise to a $\mathbb{Z}^{2}$-grading on it.

Theorem 5.1 implies the following result, which will be very useful for computing explicitly the Auslander-Reiten quiver of $\mathcal{D}^{b}(\Lambda)$.

COROLlary 5.3. There exists a $k$-linear equivalence

$$
\mathcal{D}^{b}(\Lambda) \cong \mathcal{D}^{b}(\operatorname{Cov}(H, \partial)) / \mathbb{S}_{2}\langle 1\rangle_{\partial}
$$

Proof. By Theorem 5.1, we deduce that there is a $k$-linear equivalence between the orbit categories

$$
\mathcal{D}^{b}(\operatorname{Cov}(\Lambda, \delta)) /\langle 1\rangle_{\delta} \cong \mathcal{D}^{b}(\operatorname{Cov}(H, \partial)) / \mathbb{S}_{2}\langle 1\rangle_{\partial}
$$

Now we can use the following result due to Keller. 
Theorem 5.4 ([K2, Theorem 1]). Let $Q_{H}$ be an acyclic quiver, and let $\partial$ be a $\mathbb{Z}$-grading on $Q_{H}$. Let $F:=-\otimes_{\operatorname{Cov}(H, \partial)}^{L} X$ be an autoequivalence of $\mathcal{D}^{b}(\operatorname{Cov}(H, \partial))$ for some object $X \in \mathcal{D}(\operatorname{Cov}(H, \partial))$. Assume that

(1) for each indecomposable $U \in \bmod \operatorname{Cov}(H, \partial)$, the set $\left\{i \in \mathbb{Z} \mid F^{i} U \in\right.$ $\bmod \operatorname{Cov}(H, \partial)\}$ is finite;

(2) there exists $N \geq 0$ such that for each indecomposable $X \in \mathcal{D}^{b}(\operatorname{Cov}(H, \partial))$, there exists $0 \leq n \leq N$ and $i \in \mathbb{Z}$ with $F^{i} X[-n] \in \bmod \operatorname{Cov}(H, \partial)$.

Then the orbit category $\mathcal{D}^{b}(\operatorname{Cov}(H, \partial)) / F$ admits a natural triangulated structure such that the projection functor $\mathcal{D}^{b}(\operatorname{Cov}(H, \partial)) \rightarrow \mathcal{D}^{b}(\operatorname{Cov}(H, \partial)) / F$ is triangulated.

It is already shown in [BMRRT, Section 1] that the functor $\mathbb{S}_{2}$ satisfies conditions (1) and (2). Since the functor $\langle 1\rangle_{\partial}$ is an autoequivalence of $\bmod \operatorname{Cov}(H, \partial)$, then again the functor $\mathbb{S}_{2}\langle 1\rangle_{\partial}$ clearly satisfies conditions (1) and (2). Thus, the orbit category $\left(\mathcal{D}^{b}(\operatorname{Cov}(\Lambda, \delta)) /\langle 1\rangle_{\delta}\right) \cong \mathcal{D}^{b}(\operatorname{Cov}(H, \partial)) / \mathbb{S}_{2}\langle 1\rangle_{\partial}$ is triangulated, and the natural functor

$$
\mathcal{D}^{b}(\operatorname{Cov}(\Lambda, \delta)) \longrightarrow \mathcal{D}^{b}(\operatorname{Cov}(\Lambda, \delta)) /\langle 1\rangle_{\delta}
$$

is a triangle functor.

By [AO1, Corollary 7.14], the derived category $\mathcal{D}^{b}(\Lambda)$ is equivalent to the triangulated hull $\left(\mathcal{D}^{b}(\operatorname{Cov}(\Lambda, \delta)) /\langle 1\rangle_{\delta}\right)_{\Delta}$. Hence, we have the following commutative diagram:

$$
\mathcal{D}^{b}(\operatorname{Cov}(\Lambda, \delta)) \stackrel{\stackrel{A}{\longrightarrow} \mathcal{D}^{b}(\operatorname{Cov}(\Lambda, \delta)) /\langle 1\rangle \stackrel{B}{\longrightarrow}}{\longrightarrow} \mathcal{D}^{b}(\Lambda),
$$

where $A$ and $C$ are triangle functors. Therefore, $B$ commutes with the shift and sends triangles to triangles. Hence, the fully faithful functor $B$ is a $k$-equivalence

$$
\mathcal{D}^{b}\left(\operatorname{Cov}(\Lambda, \delta) /\langle 1\rangle_{\delta}\right) \cong \mathcal{D}^{b}(\operatorname{Cov}(H, \partial)) / \mathbb{S}_{2}\langle 1\rangle_{\partial}
$$

\subsection{The shape of the Auslander-Reiten quiver of $\mathcal{D}^{b}(\Lambda)$}

In this section, we use Corollary 5.3 to compute explicitly the shape of the Auslander-Reiten quiver of the derived category of an algebra of cluster type $\widetilde{A}_{p, q}$. 
Throughout this section, $\Lambda$ is an algebra of cluster type $\widetilde{A}_{p, q}$ and of weight $w \neq 0$. Let $\partial$ be a grading induced by $\Lambda$ on $Q_{H}$. By Lemma 4.2 , we can assume that $\partial$ is the following grading:

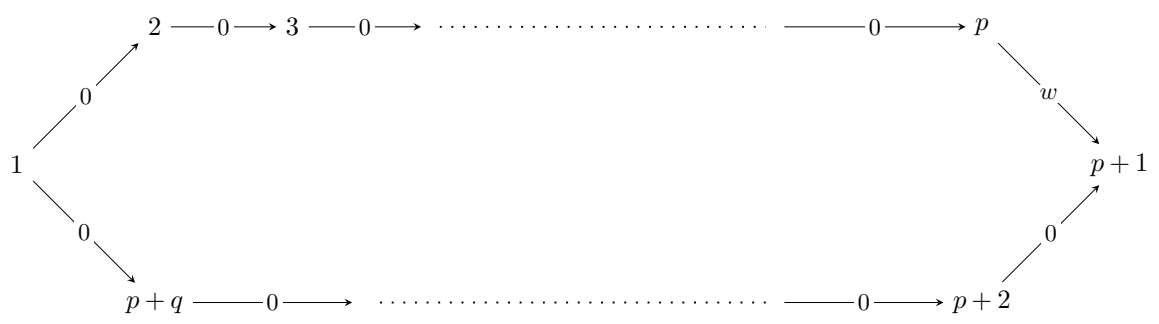

By Corollary 5.3, we have a $k$-linear equivalence

$$
\mathcal{D}^{b}(\Lambda) \cong \mathcal{D}^{b}(\operatorname{Cov}(H, \partial)) / \mathbb{S}_{2}\langle 1\rangle_{\partial}
$$

The quiver of the subcategory $\operatorname{Cov}(H, \partial)=\operatorname{add}\left\{H\langle p\rangle_{\partial}, p \in \mathbb{Z}\right\} \subset$ gr $H$ consists of $|w|$ connected components of type $A_{\infty}^{\infty}$ with the following orientation:

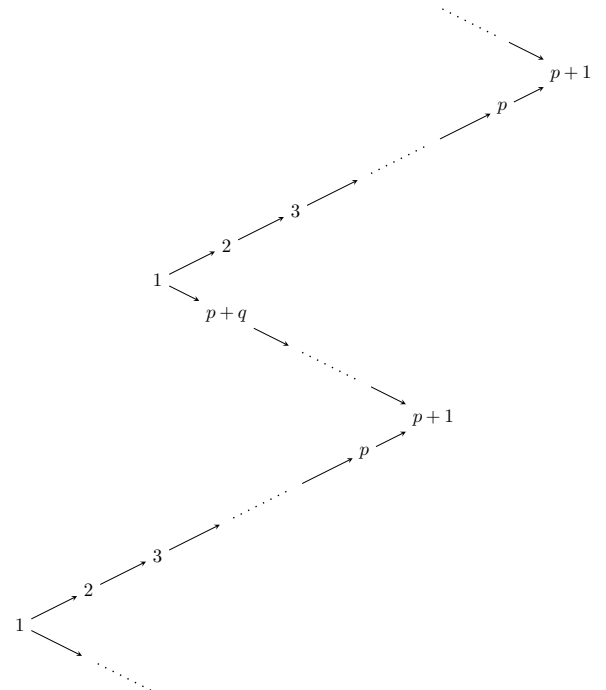

Then it is not hard to compute the Auslander-Reiten quiver of the module category $\bmod \operatorname{Cov}(H, \partial)$. It has $4|w|$ connected components: $\mathcal{P}_{i}, \mathcal{R}_{i}^{p}, \mathcal{R}_{i}^{q}$, and $\mathcal{I}_{i}$, with $i \in \mathbb{Z} / w \mathbb{Z}$ satisfying $\mathcal{P}_{i}=\mathcal{P}_{0}\langle i\rangle, \mathcal{R}_{i}^{p}=\mathcal{R}_{0}^{p}\langle i\rangle, \mathcal{R}_{i}^{q}=\mathcal{R}_{0}^{q}\langle i\rangle$, and $\mathcal{I}_{i}=\mathcal{I}_{0}\langle i\rangle$ for any $i \in \mathbb{Z} / w \mathbb{Z}$.

The component $\mathcal{P}_{0}$ is of type $\mathbb{N} A_{\infty}^{\infty}$ and contains the indecomposable projective modules $P_{j}\langle 0\rangle$ for $j=1, \ldots, p+q$. Its shape is described in Figure 5.1. 


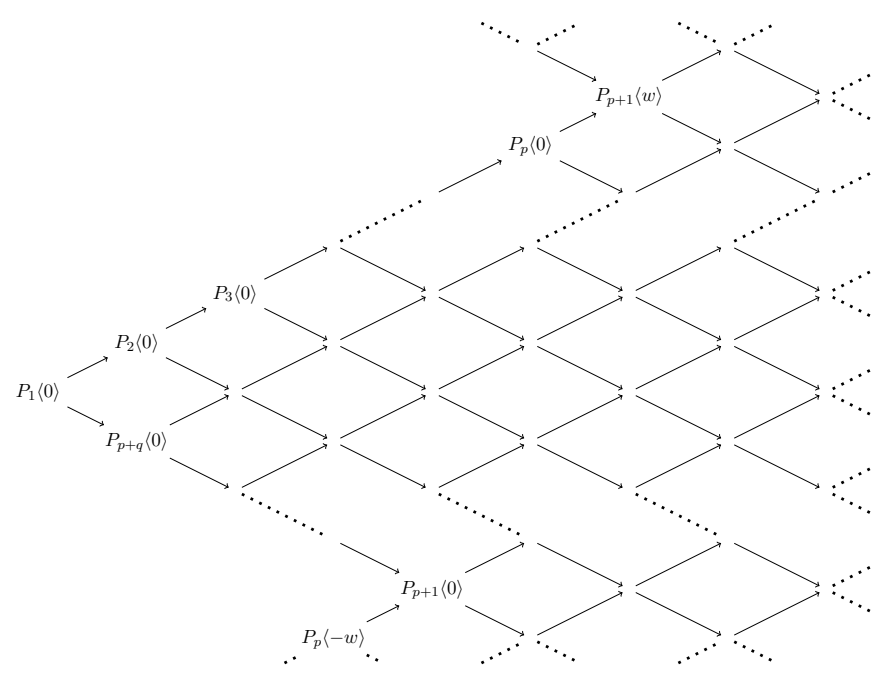

Figure 5.1: Shape of the component $\mathcal{P}_{0}$ of the Auslander-Reiten quiver of $\bmod (\operatorname{Cov}(H, \partial))$.

The component $\mathcal{I}_{0}$ is of type $(-\mathbb{N}) A_{\infty}^{\infty}$ and contains the indecomposable injective modules $I_{j}\langle 0\rangle$ for $j=1, \ldots, p+q$. Its shape is described in Figure 5.2 .

The components $\mathcal{R}_{0}^{p}$ and $\mathcal{R}_{0}^{q}$ are of type $\mathbb{Z} A_{\infty}$ and contain the regular modules. The shape of the connected components $\mathcal{R}_{0}^{p}$ and $\mathcal{R}_{0}^{q}$ is described in Figures 5.3 and 5.4. In these figures, $S_{i}\langle m\rangle$ is the $m$-degree shift of the simple module associated with the vertex $i$, the object $M\langle 0\rangle$ is defined by the exact sequepce $P_{p}\langle-w\rangle \stackrel{a_{p}}{\longrightarrow} P_{p+1}\langle 0\rangle \longrightarrow M\langle 0\rangle \longrightarrow 0$,

and the object $N\langle 0\rangle$ is defined by the exact sequence

$$
P_{p+2}\langle 0\rangle \stackrel{b_{q}}{\longrightarrow} P_{p+1}\langle 0\rangle \longrightarrow N\langle 0\rangle \longrightarrow 0 .
$$

Under the forgetful functor $\bmod \operatorname{Cov}(H, \partial) \rightarrow \bmod H$, the components $\mathcal{P}_{i}$ are sent to $\mathcal{P}$ the preprojective component of $\bmod H$, the components $\mathcal{I}_{i}$ are sent to the preinjective component $\mathcal{I}$, the components $\mathcal{R}_{j}^{p}$ are sent to the exceptional tube of rank $p$, and the components $\mathcal{R}_{j}^{q}$ are sent to the exceptional tube of $\operatorname{rank} q$. The indecomposable $H$-modules lying in the 


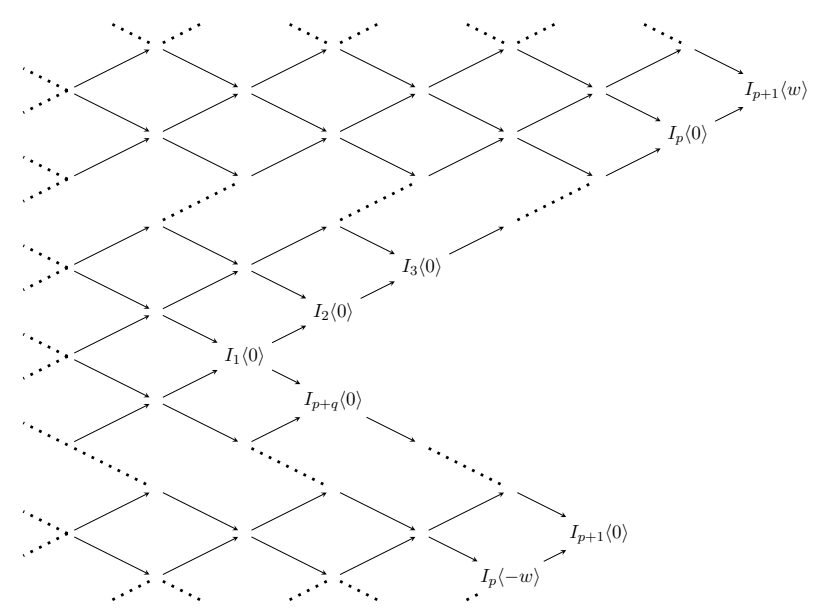

Figure 5.2: Shape of the component $\mathcal{I}_{0}$ of the Auslander-Reiten quiver of $\bmod (\operatorname{Cov}(H, \partial))$.

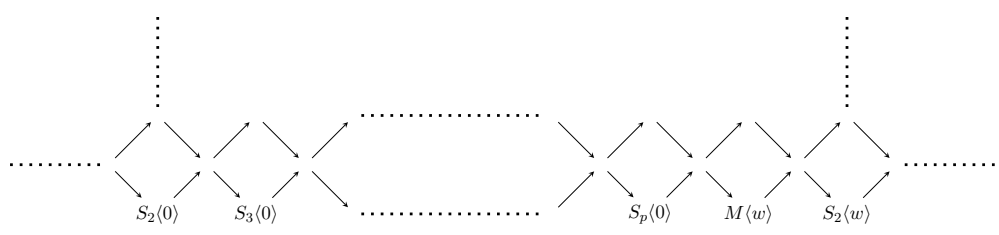

Figure 5.3: Shape of the connected component $\mathcal{R}_{0}^{p}$.

homogeneous tubes are not gradable if $w \neq 0$; therefore, the description above is complete.

Since $\bmod \operatorname{Cov}(H, \partial)$ is a hereditary category, one can easily deduce the shape of the Auslander-Reiten quiver of $\mathcal{D}^{b}(\operatorname{Cov}(H, \partial))$. It has three kinds of components, $\mathcal{P}_{(i, n)}, \mathcal{R}_{(i, n)}^{p}$, and $\mathcal{R}_{(i, n)}^{q}$, with $i \in \mathbb{Z} / w \mathbb{Z}$ and $n \in \mathbb{Z}$. The component $\mathcal{P}_{(0,0)}$ contains $\mathcal{P}_{0}$ and $\mathcal{I}_{0}[-1]$ and is described in Figure 5.5. The component $\mathcal{R}_{(0,0)}^{p}$ (resp., $\mathcal{R}_{(0,0)}^{q}$ ) is $\mathcal{R}_{0}^{p}$ (resp., $\mathcal{R}_{0}^{q}$ ). Moreover, we have $\mathcal{P}_{(i, n)}=$ $\mathcal{P}_{(0,0)}\langle i\rangle[n], \mathcal{R}_{(i, n)}^{p}=\mathcal{R}_{(0,0)}^{p}\langle i\rangle[n]$, and $\mathcal{R}_{(i, n)}^{q}=\mathcal{R}_{(0,0)}^{q}\langle i\rangle[n]$ for $i \in \mathbb{Z} / w \mathbb{Z}$ and $n \in \mathbb{Z}$. 


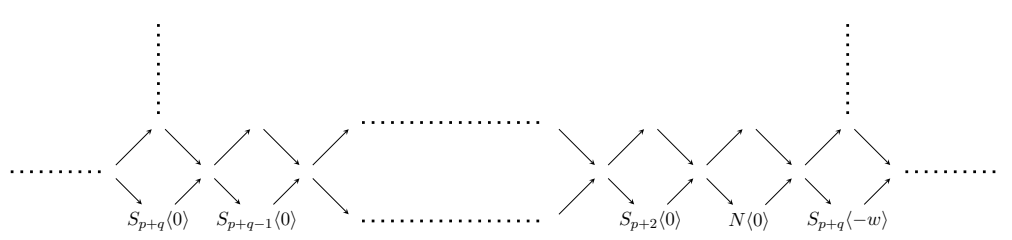

Figure 5.4: Shape of the connected component $\mathcal{R}_{0}^{q}$.

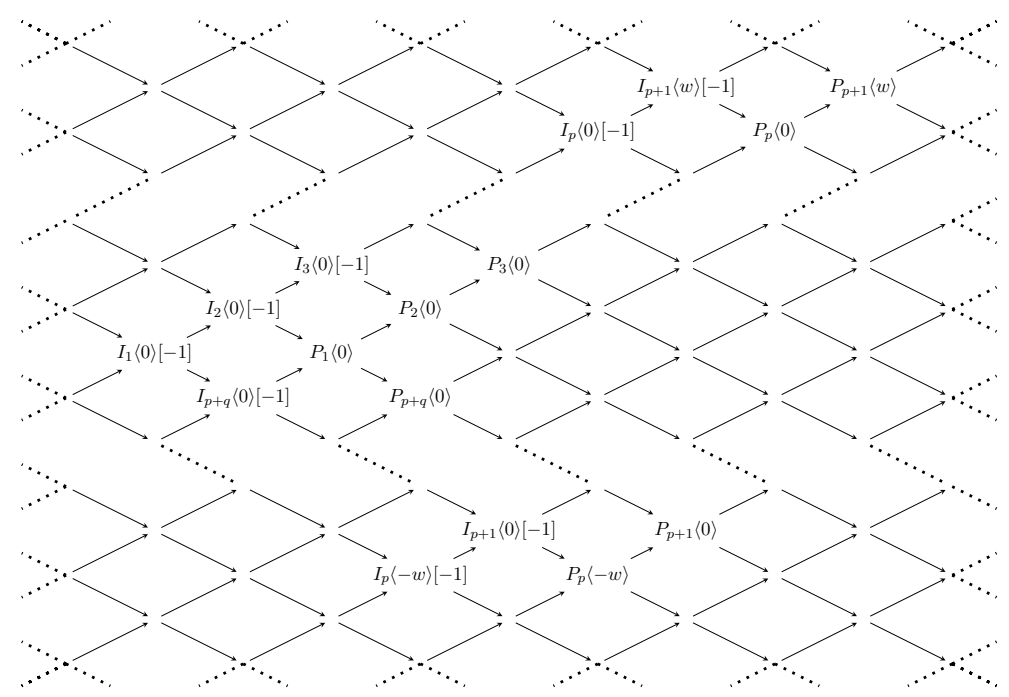

Figure 5.5: Shape of the component $\mathcal{P}_{(0,0)}$ of the Auslander-Reiten quiver of $\mathcal{D}^{b}(\operatorname{Cov}(H, \partial))$.

The morphisms satisfy the following:

$$
\begin{array}{ll}
\operatorname{Hom}\left(\mathcal{P}_{(i, n)}, \mathcal{P}_{(j, m)}\right) \neq 0 & \text { if and only if } i=j \text { and } m \in\{n, n+1\} \\
\operatorname{Hom}\left(\mathcal{P}_{(i, n)}, \mathcal{R}_{(j, m)}^{p, q}\right) \neq 0 & \text { if and only if } i=j \text { and } m=n, \\
\operatorname{Hom}\left(\mathcal{R}_{(i, n)}^{p, q}, \mathcal{P}_{(j, m)}\right) \neq 0 & \text { if and only if } i=j \text { and } m=n+1, \\
\operatorname{Hom}\left(\mathcal{R}_{(i, n)}^{p, q}, \mathcal{R}_{(j, m)}^{p, q}\right) \neq 0 & \text { if and only if } \quad i=j \text { and } m \in\{n, n+1\}
\end{array}
$$




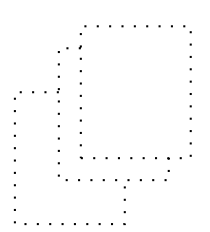

$\mathcal{P}_{(i, 0)}$

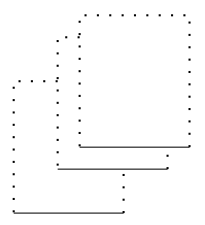

$\mathcal{R}_{(i, 0)}^{p}$

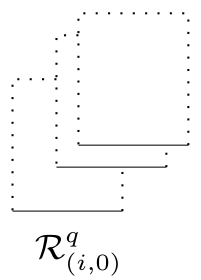

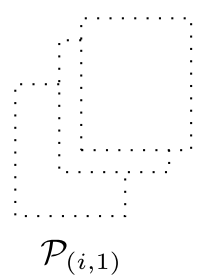

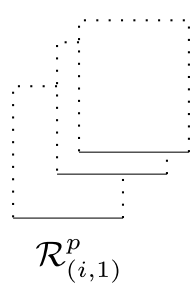

Figure 5.6: Auslander-Reiten quiver of $\mathcal{D}^{b}(\operatorname{Cov}(H, \partial))$.

Now we can compute the Auslander-Reiten quiver of the orbit category $\mathcal{D}^{b}(\operatorname{Cov}(H, \partial)) / \mathbb{S}_{2}\langle 1\rangle$. We look at the action of the functor $\mathbb{S}_{2}\langle 1\rangle$ on the connected components of the Auslander-Reiten quiver of $\mathcal{D}^{b}(\operatorname{Cov}(H, \partial))$ (See Figure 5.6). The functor $\mathbb{S}_{2}\langle 1\rangle$ acts on the set $\left\{\mathcal{P}_{(i, n)}, i \in \mathbb{Z} / w \mathbb{Z}, n \in\right.$ $\mathbb{Z}\}$ by

$$
\mathbb{S}_{2}\left(\mathcal{P}_{(i, n)}\right)\langle 1\rangle=\tau\left(\mathcal{P}_{(i, n)}\right)[-1]\langle 1\rangle=\mathcal{P}_{(i, n)}[-1]\langle 1\rangle=\mathcal{P}_{(i+1, n-1)}
$$

Then there are exactly $|w|$ orbits which are $\left(\mathbb{S}_{2}\langle 1\rangle\right)^{\mathbb{Z}} \cdot \mathcal{P}_{(i, 0)}$ for $i \in \mathbb{Z} / w \mathbb{Z}$. Similarly, $\mathbb{S}_{2}\langle 1\rangle$ acts on the sets $\left\{\mathcal{R}_{(i, n)}^{p}, i \in \mathbb{Z} / w \mathbb{Z}, n \in \mathbb{Z}\right\}$ and $\left\{\mathcal{R}_{(i, n)}^{q}, i \in\right.$ $\mathbb{Z} / w \mathbb{Z}, n \in \mathbb{Z}\}$ by

$$
\mathbb{S}_{2}\left(\mathcal{R}_{(i, n)}^{p}\right)\langle 1\rangle=\mathcal{R}_{(i+1, n-1)}^{p} \quad \text { and } \quad \mathbb{S}_{2}\left(\mathcal{R}_{(i, n)}^{q}\right)\langle 1\rangle=\mathcal{R}_{(i+1, n-1)}^{q} .
$$

Therefore, we get $2|w|$ orbits which are $\left(\mathbb{S}_{2}\langle 1\rangle\right)^{\mathbb{Z}} \cdot \mathcal{R}_{(i, 0)}^{p}$ and $\left(\mathbb{S}_{2}\langle 1\rangle\right)^{\mathbb{Z}} \cdot \mathcal{R}_{(i, 0)}^{q}$ for $i \in \mathbb{Z} / w \mathbb{Z}$.

Finally, the shape of the Auslander-Reiten quiver of $\mathcal{D}^{b}(\operatorname{Cov}(H, \partial)) / \mathbb{S}_{2}\langle 1\rangle$ is described in Figure 5.7. This description can be summarized in the following.

COROllary 5.5. Let $\Lambda$ be an algebra of cluster type $\widetilde{A}_{p, q}$ and of weight $w \neq 0$. Then the Auslander-Reiten quiver of $\mathcal{D}^{b}(\Lambda)$ has exactly $3|w|$ connected components:

- $|w|$ components of type $\mathbb{Z} A_{\infty}^{\infty}$,

- $2|w|$ components of type $\mathbb{Z} A_{\infty}$.

\subsection{Consequences of the description of the Auslander-Reiten quiver}

From this description we also get the following consequences. 


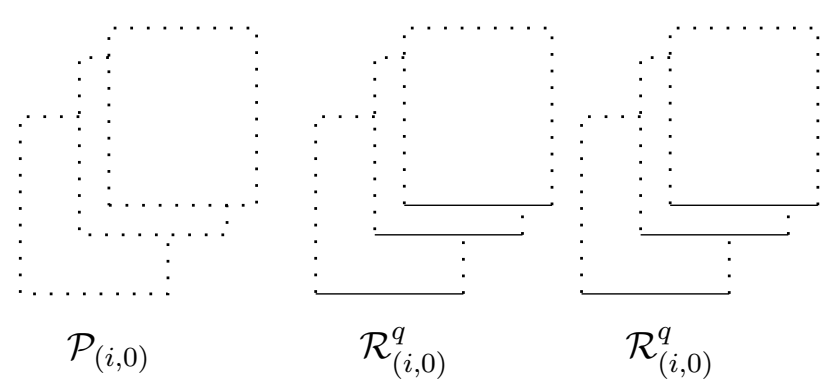

Figure 5.7: Auslander-Reiten quiver of the category $\mathcal{D}^{b} \Lambda$, where $\Lambda$ is of cluster type $\widetilde{A}_{p, q}$ and of weight $w>0$.

Corollary 5.6. Let $\Lambda$ be an algebra of cluster type $\widetilde{A}_{p, q}$ and of weight $w \neq 0$. Then $\Lambda$ is representation-finite.

Proof. The algebra $\Lambda$ is derived discrete in the sense of $[\mathrm{V}]$. Hence, it is representation-finite.

REMARK 5.7. Let $\Lambda$ be an algebra of cluster type $\widetilde{A}_{p, q}$ and of weight $w \neq 0$. Then the category $\mathcal{D}^{b}(\Lambda)$ is locally fractionally Calabi-Yau of dimensions $(p-2 w) /(p-w)$ and $(q+2 w) /(q+w)$ in the sense that there exist objects $X$ and $Y$ such that there are isomorphisms $\mathbb{S}^{p-w} X \cong X[p-2 w]$ and $\mathbb{S}^{q+w} Y \cong Y[q+2 w]$. Such algebras are studied in [AO2, Section 6].

From Section 5.2, we also deduce a result for the image of the derived category in the cluster category.

Corollary 5.8. Let $\Lambda$ be an algebra of cluster type $\widetilde{A}_{p, q}$ and of weight $w \neq 0$. Then the quiver of the orbit category $\mathcal{D}^{b}(\Lambda) / \mathbb{S}_{2}$ has three connected components which are of the forms $\mathbb{Z} \widetilde{A}_{p, q}, \mathbb{Z} A_{\infty} /\left(\tau^{p}\right)$, and $\mathbb{Z} A_{\infty} /\left(\tau^{q}\right)$. 
Proof. From Corollary 5.3, we have the following diagram:

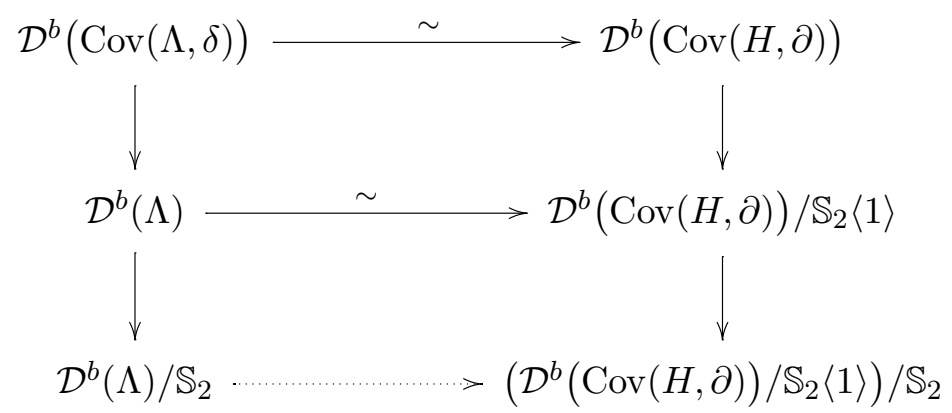

The upper functor is a triangle functor; hence, it commutes with $\mathbb{S}_{2}$. Therefore, the $k$-equivalence of Corollary 5.3 commutes with $\mathbb{S}_{2}$. Using this, we deduce that we have a $k$-equivalence

$$
\mathcal{D}^{b}(\Lambda) / \mathbb{S}_{2} \longrightarrow\left(\mathcal{D}^{b}(\operatorname{Cov}(H, \partial)) / \mathbb{S}_{2}\langle 1\rangle\right) / \mathbb{S}_{2}
$$

Therefore, the Auslander-Reiten quiver of $\mathcal{D}^{b}(\Lambda) / \mathbb{S}_{2}$ is the same as the Auslander-Reiten quiver of the orbit category $\left(\mathcal{D}^{b}(\operatorname{Cov}(H, \partial)) / \mathbb{S}_{2}\langle 1\rangle\right) / \mathbb{S}_{2}$. Note that since $\mathbb{S}_{2} \cong\langle-1\rangle$ in $\left(\mathcal{D}^{b}(\operatorname{Cov}(H, \partial)) / \mathbb{S}_{2}\langle 1\rangle\right)$, we just have to understand the action of $\langle 1\rangle$ in the category $\left(\mathcal{D}^{b}(\operatorname{Cov}(H, \partial)) / \mathbb{S}_{2}\langle 1\rangle\right)$. We know that the category $\left(\mathcal{D}^{b}(\operatorname{Cov}(H, \partial)) / \mathbb{S}_{2}\langle 1\rangle\right)$ has $3|w|$ components which are

$$
\mathcal{P}_{(i, 0)}, \mathcal{R}_{(i, 0)}^{p}, \quad \text { and } \quad \mathcal{R}_{(i, 0)}^{q}, \quad \text { for } i \in \mathbb{Z} / w \mathbb{Z} .
$$

Moreover, we have the equalities

$$
\begin{aligned}
& \mathcal{P}_{(i, 0)}\langle 1\rangle=\mathcal{P}_{(i+1,0)}, \quad \mathcal{R}_{(i, 0)}^{p}\langle 1\rangle=\mathcal{R}_{(i+1,0)}^{p}, \quad \text { and } \\
& \mathcal{R}_{(i, 0)}^{q}\langle 1\rangle=\mathcal{R}_{(i+1,0)}^{q} .
\end{aligned}
$$

Thus, for each connected component $\Gamma$ of the Auslander-Reiten quiver of $\left(\mathcal{D}^{b}(\operatorname{Cov}(H, \partial)) / \mathbb{S}_{2}\langle 1\rangle\right)$, we have $\Gamma\langle w\rangle=\Gamma$. Now it is easy to check that for $X \in \mathcal{R}_{(i, 0)}$ we have $X\langle w\rangle \cong \tau^{-p} X$. The action of $\langle w\rangle$ on the component $\mathcal{P}_{(0,0)}$ makes $\mathcal{P}_{(0,0)} /\langle w\rangle$ isomorphic to $\mathbb{Z} \widetilde{A}_{p, q}$. Therefore, we get the result.

\section{$\S 6$. Explicit description of algebras of cluster type $\widetilde{A}_{p, q}$}

In this section, we explicitly describe (in terms of quivers with relations) the algebras of cluster type $\widetilde{A}_{p, q}$, and we give an easy method for computing 
the weight of such an algebra. The strategy consists of first describing the cluster-tilted algebras of type $\widetilde{A}_{p, q}$, following [Ba], and then showing that all algebras of cluster type $\widetilde{A}_{p, q}$ can been seen as the degree 0 part of a cluster-tilted algebra of type $\widetilde{A}_{p, q}$ with an appropriate grading. We start by describing the algebras of cluster type $A_{n}$ using a similar approach.

\subsection{Algebras of cluster type $A_{n}$}

The aim of this section is to describe all algebras of global dimension at most 2 which are of cluster type $A_{n}$. By Corollary 3.16, we already know that they are the algebras of global dimension at most 2 derived equivalent to $k Q$, where $Q$ is a quiver of type $A_{n}$. A description of iterated-tilted algebras of type $A_{n}$ was done by Assem [Am]. Here we use other techniques, based on further developments in cluster-tilting theory.

We start with some definitions.

Definition 6.1. Let $Q$ be a quiver. A cycle $a_{1} \cdots a_{r}$ in $Q$ is called irreducible if for all $1 \leq i \neq j \leq r$ we have $s\left(a_{i}\right) \neq s\left(a_{j}\right)$. All cycles which are not irreducible are called reducible.

Definition 6.2. For $n \geq 1$, we define the class $\mathcal{M}_{n}^{A}$ of quivers $Q$ that satisfy the following.

- They have $n$ vertices.

- All nontrivial irreducible cycles are oriented and of length 3.

- A vertex has valency at most 4 .

- If a vertex has valency 4 , then two of its adjacent arrows belong to one 3-cycle, and the other two belong to another 3-cycle.

- If a vertex has valency 3 , then two of its adjacent arrows belong to a 3-cycle, and the third arrow does not belong to any 3-cycle.

The set $\mathcal{M}^{A}$ denotes the union of all $\mathcal{M}_{n}^{A}$. For a quiver $Q$ in $\mathcal{M}_{A}$, we denote by $W_{Q}$ the sum of all oriented 3-cycles. This is a potential in the sense of [DWZ].

Theorem 6.3 ([BV, Proposition 2.4]). Let $\Gamma$ be a basic finite-dimensional algebra. Then $\Gamma$ is a cluster-tilted algebra of type $A_{n}$ if and only if there exists a quiver $Q$ in $\mathcal{M}_{n}^{A}$ such that we have an isomorphism $\Gamma \cong \operatorname{Jac}\left(Q, W_{Q}\right)$.

The main result of this section is the following.

TheOREM 6.4. Let $Q$ be a quiver in $\mathcal{M}_{n}^{A}$, and let $d$ be a $W_{Q^{-g} \text {-gading (see }}$ Definition 3.10). It yields a grading on the Jacobian algebra $B:=\operatorname{Jac}(Q, W)$. Denote by $\Lambda:=B_{0}$ its degree 0 part. Then $\Lambda$ is an algebra of global dimension 
at most 2 and of cluster type $A_{n}$. Moreover, each basic algebra of global dimension at most 2 and of cluster type $A_{n}$ is isomorphic to such a $\Lambda$.

Proof. We divide the proof into several steps for the convenience of the reader.

Step 1: The global dimension of the algebra $\Lambda$ is at most 2 .

Denote by $Q^{(0)}$ the subquiver of $Q$ which is defined by $Q_{0}^{(0)}:=Q_{0}$ and $Q_{1}^{(0)}:=\left\{a \in Q_{1}, d(a)=0\right\}$. Then we have

$$
\Lambda \cong k Q^{(0)} /\left\langle\partial_{a} W_{Q}, d(a)=1\right\rangle .
$$

Note that since $W$ is a sum of 3-cycles, the ideal of relations $\left\langle\partial_{a} W_{Q}, d(a)=1\right\rangle$ is contained in $k Q_{2}^{(0)}$; that is, every relation is of length 2 . Let $i \in Q_{0}$ be a vertex, and let $e_{i}$ be the associated primitive idempotent of $\Lambda$. Then the projective resolution of the simple $\Lambda$-module associated to $i$ is given by

$$
\begin{gathered}
\bigoplus_{b, s(b)=i, d(b)=1} e_{t(b)} \Lambda \stackrel{\left(f_{a, b}\right)}{\longrightarrow} \bigoplus_{a, t(a)=i, d(a)=0} e_{s(a)} \Lambda \\
\longrightarrow e_{i} \Lambda \longrightarrow S_{i} \longrightarrow 0 .
\end{gathered}
$$

Let $b: i \rightarrow l$ be an arrow of degree 1 , and let $a: j \rightarrow i$ such that $f_{a, b} \neq 0$. Then there exists a 3-cycle

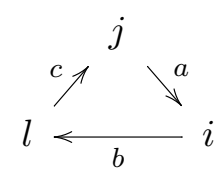

and the map $f_{a, b}: e_{l} \Lambda \rightarrow e_{j} \Lambda$ is induced by $c$. The arrow $c$ does not belong to another 3-cycle; therefore, there is no relation between some predecessor of $l$ and $j$. Hence, the map $f_{a, b}: e_{l} \Lambda \rightarrow e_{j} \Lambda$ is a monomorphism.

Let $a$ be an arrow with $t(a)=i$ and of degree 0 . Then $a$ belongs to at most one cycle. Therefore, there exists at most one arrow $b$ of degree 1 with $s(b)=i$ such that $f_{a, b}$ does not vanish. Therefore, the map

$$
\bigoplus_{b, s(b)=i, d(b)=1} e_{t(b)} \Lambda \stackrel{\left(f_{a, b}\right)}{\longrightarrow} \bigoplus_{a, t(a)=i, d(a)=0} e_{s(a)} \Lambda
$$


is injective, and the projective dimension of $S_{i}$ is at most 2 .

Step 2: We have an isomorphism $\operatorname{Jac}\left(Q, W_{Q}\right) \cong \operatorname{End}_{\mathcal{C}_{\Lambda}}(\pi \Lambda)$.

Since $d$ is a $W_{Q}$-grading, the set $\left\{\partial_{a} W_{Q}, d(a)=1\right\}$ is a set of minimal relations for $\Lambda$. Therefore, by Theorem 2.5, we have $\bar{Q}^{(0)}=Q$ and $W_{\Lambda}=W_{Q}$ and hence Step 2.

Step 3: There is a triangle equivalence $\mathcal{C}_{\Lambda} \cong \mathcal{C}_{A_{n}}$.

By Theorems 6.3, 2.9, and 2.7, there exists a sequence of mutation $s$ such that $\mu_{s}\left(Q, W_{Q}\right)=\left(Q_{H}, 0\right)$. Therefore, we are done by Corollary 3.2 .

Step 4: Each algebra of cluster type $A_{n}$ and of global dimension at most 2 is isomorphic to the degree 0 part of a graded Jacobian algebra $\operatorname{Jac}\left(Q, W_{Q}, d\right)$ where $Q \in \mathcal{M}_{n}^{A}$ and $d$ is a $W_{Q}$-grading.

Let $\Lambda \cong k Q_{\Lambda} / I$ be an algebra of global dimension at most 2 and of cluster type $A_{n}$. Denote by $f: \mathcal{C}_{\Lambda} \rightarrow \mathcal{C}_{Q}$ the triangle equivalence. By Proposition 3.11, there exists a graded $\mathrm{QP}\left(Q_{\bar{\Lambda}}, W, d\right)$, where $W$ is rigid and $d$ is a $W$-grading, such that we have

$$
\bar{\Lambda} \underset{\mathbb{Z}}{\cong} \operatorname{Jac}\left(Q_{\bar{\Lambda}}, W, d\right)
$$

Now the object $f\left(\pi_{\Lambda}(\Lambda)\right)$ is a cluster-tilting object in $\mathcal{C}_{A_{n}}$. Thus, by Theorem 6.3, there exists $Q \in \mathcal{M}_{n}^{A}$ such that we have an isomorphism

$$
\bar{\Lambda} \cong \operatorname{Jac}\left(Q, W_{Q}\right)
$$

It is clear that we have $Q=Q_{\bar{\Lambda}}$. We then conclude using the following lemma.

Lemma 6.5. Let $(Q, W, d)$ be a graded $Q P$ where $Q \in \mathcal{M}_{n}^{A}$ and $W$ is rigid. Then $d$ makes $W_{Q}$ homogeneous of degree 1 , and we have an isomorphism of $\mathbb{Z}$-graded algebras

$$
\operatorname{Jac}\left(Q, W_{Q}, d\right) \cong \underset{\mathbb{Z}}{ } \operatorname{Jac}(Q, W, d) .
$$

Proof. Denote by $C_{1}, \ldots, C_{l}$ the oriented 3 -cycles of $Q$, so that we have $W_{Q}=\sum_{i=1}^{r} C_{i}$. One easily checks that the irreducible oriented cycles of the quiver $Q$ are exactly the $C_{i}$ (up to cyclic equivalence). Therefore, we can assume that

$$
W=\sum_{i=1}^{r} \lambda_{i} C_{i}+\text { extra terms, }
$$

where the extra terms are reducible. If there exists $i$ with $\lambda_{i}=0$, then the cycle $C_{i}$ and all the cycles cyclically equivalent to it are not in the Jacobian 
ideal since there are no cycles of length at most 2 in the quiver $Q$. Therefore, since $W$ is rigid, we have $\lambda_{i} \neq 0$ for all $i=1, \ldots, r$. Now the existence of the grading $d$ implies that $d\left(C_{i}\right)=1$ for all $i=1, \ldots, r$, so any reducible cycle is of degree at least 2. Hence, the extra terms in the potential have to be zero, and we have $W=\sum_{i=1}^{r} \lambda_{i} C_{i}$ with $\lambda_{i} \neq 0$.

Remark 6.6. Note that Theorem 6.4 is not true for the other Dynkin types. Let $(Q, d)$ be the graded quiver

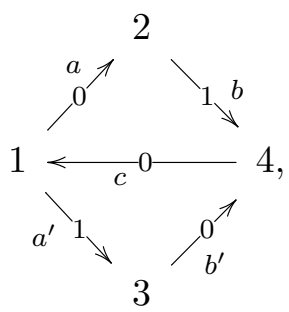

and let $W:=c b a+c b^{\prime} a^{\prime}$. The Jacobian algebra $\operatorname{Jac}(Q, W)$ is a cluster-tilted algebra of type $D_{4}$. The grading $d$ is a $W$-grading, but the degree 0 part of the graded algebra $\operatorname{Jac}(Q, W, d)$ is an iterated-tilted algebra of type $A_{4}$ and of global dimension 3 , so it cannot be of cluster type $D_{4}$.

\subsection{Cluster-tilted algebras of type $\widetilde{A}_{p, q}$}

Definition 6.7 ([Ba, Definition 3.3]). For $p \geq q \geq 1$, we define the class $\mathcal{M}_{p, q}^{\widetilde{A}}$ of quivers $Q$ that satisfy the following conditions (see Figure 6.1).

- The quiver $Q$ has $p+q$ vertices.

- There exist integers $1 \leq p_{1}<p_{2}<\cdots<p_{r} \leq p$ and $1 \leq q_{1}<q_{2}<\cdots<$ $q_{r} \leq q$ such that $Q$ contains precisely one full subquiver $C$ which is a nonoriented cycle of type $\left(p_{1}, q_{1}, p_{2}-p_{1}, q_{2}-q_{1}, \ldots, p_{r}-p_{r-1}, q_{r}-q_{r-1}\right)$. (That is, $C$ is the composition of $p_{1}$ arrows going in one direction with $q_{1}$ arrows going in the other direction, with $p_{2}-p_{1}$ arrows going the first direction, etc.) We denote by $a_{1}, \ldots, a_{p_{r}}$ the arrows of $C$ going in one direction, and we call them the p-arrows. We denote by $b_{1}, \ldots, b_{q_{r}}$ the arrows going in the opposite direction, and we call them the $q$-arrows.

- Each arrow connecting $C$ to a vertex not in $C$ is in exactly one 3-cycle of $Q$ of the form

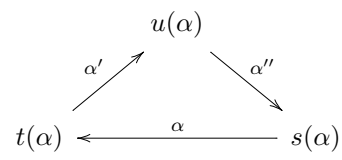




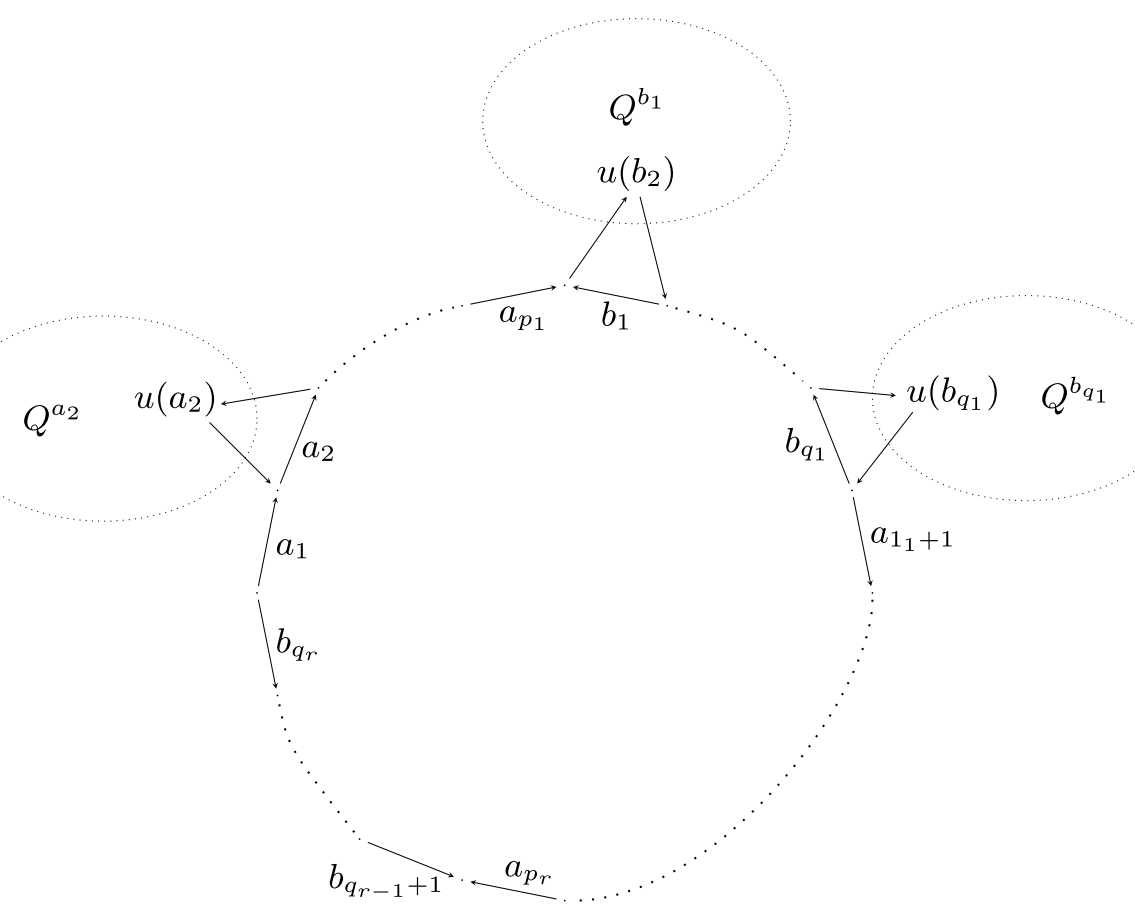

Figure 6.1: Shape of a quiver in $\mathcal{M}_{p, q}^{\widetilde{A}}$.

where $\alpha$ is in $C$. We denote by $u(\alpha)$ the connecting vertex. It has valency at most 4 . When its valency is 4 , the adjacent arrows which are not $\alpha^{\prime}$ and $\alpha^{\prime \prime}$ belong to exactly one 3 -cycle, and when it has valency 3 , the third arrow does not belong to any 3 -cycle. Moreover, the subquiver containing $C, \alpha^{\prime}$, and $\alpha^{\prime \prime}$ is a full subquiver of $Q$. Hence, we cannot have $u(\alpha)=u(\beta)$ for $\alpha \neq \beta$.

- The full subquiver of $Q$ whose vertices are not in $C$ is a disjoint union of quivers $Q^{\alpha} \in \mathcal{M}^{A}$, where $\alpha$ is an arrow of the nonoriented cycle. The quiver $Q^{\alpha}$ is empty if there is no 3-cycle containing $\alpha$, and the quiver $Q^{\alpha}$ contains the vertex $u(\alpha)$ if there is a 3-cycle

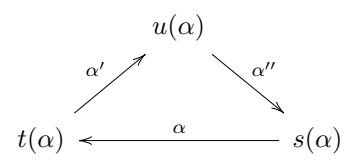


- We have the equalities

$$
p=\sum_{l=1}^{p_{r}} \sharp Q_{0}^{a_{l}}+p_{r} \quad \text { and } \quad q=\sum_{l=1}^{q_{r}} \sharp Q_{0}^{b_{l}}+q_{r} .
$$

For a quiver $Q$ in $\mathcal{M}_{p, q}^{\widetilde{A}}$ (see Figure 6.1) we denote by $W_{Q}$ the sum of all oriented 3-cycles. More precisely, we define

$$
W_{Q}:=\sum_{\alpha \in C}\left(\alpha^{\prime \prime} \alpha^{\prime} \alpha+W_{Q^{\alpha}}\right)
$$

where $C$ is the nonoriented cycle of $Q$. This is a rigid potential in the sense of $[\mathrm{DWZ}]$.

TheOREM 6.8 ([Ba, Section 3]). Let $\Gamma$ be a finite-dimensional algebra. Then $\Gamma$ is a cluster-tilted algebra of type $\widetilde{A}_{p, q}$ if and only if there exists a quiver $Q$ in $\mathcal{M}_{p, q}^{\widetilde{A}}$ such that we have an isomorphism

$$
\Gamma \cong \operatorname{Jac}\left(Q, W_{Q}\right) \text {. }
$$

\subsection{Algebras of cluster type $\widetilde{A}_{p, q}$}

We have the same kind of result as for the $A_{n}$ case.

THEOREM 6.9. Let $Q$ be a quiver in $\mathcal{M}_{p, q}^{\widetilde{A}}$, and let $d$ be $W_{Q}$-grading. It yields a grading on the Jacobian algebra $B:=\operatorname{Jac}\left(Q, W_{Q}\right)$. Denote by $\Lambda:=B_{0}$ its degree 0 part. Then $\Lambda$ is an algebra of global dimension at most 2 and of cluster type $\widetilde{A}_{p, q}$. Moreover, each basic algebra of global dimension at most 2 and of cluster type $\widetilde{A}_{p, q}$ is isomorphic to such a $\Lambda$.

The proof of the first assertion is exactly the same as in the proof of Theorem 6.4 (steps 1, 2, and 3). For the proof of the second assertion, we will need the following.

LEMma 6.10. Let $(Q, W, d)$ be a graded quiver with reduced potential such that

- the quiver $Q$ is in $\mathcal{M}_{p, q}^{\widetilde{A}}$,

- the potential $W$ is rigid,

- the grading $d$ is a $W$-grading.

Then there exists an algebra isomorphism $\varphi: k \hat{Q} \rightarrow k \hat{Q}$ (where $k \hat{Q}$ is the completion of the path algebra $k Q$ ) such that $\varphi$ is the identity on the vertices, and such that $\varphi\left(W_{Q}\right)$ is cyclically equivalent to $W$. Moreover, there exists a $W_{Q}$-grading $d^{\prime}$ on $Q$ such that $\varphi:\left(k \hat{Q}, d^{\prime}\right) \rightarrow(k \hat{Q}, d)$ is an isomorphism of graded algebras. 
REMARK 6.11. This means that the graded QPs $(Q, W, d)$ and $\left(Q, W_{Q}, d^{\prime}\right)$ are graded right equivalent in the sense of [AO1, Definition 6.5].

Proof. We denote by $C_{1}, \ldots, C_{l}$ the oriented cycles such that $W_{Q}=$ $\sum_{i=1}^{l} C_{i}$, we denote by $a_{1}, \ldots, a_{p_{r}}$ the $p$-arrows, and we denote by $b_{1}, \ldots, b_{q_{r}}$ the $q$-arrows of $Q$. The $C_{i}$ are irreducible cycles of $Q$, but contrary to the $A_{n}$-case, there might be other irreducible cycles in the quiver $Q$. We treat here the most complicated case.

Assume that for all $i=1, \ldots, p_{r}$ and all $j=1, \ldots, q_{r}$, we have $Q^{a_{i}} \neq \varnothing$ and $Q^{b_{j}} \neq \varnothing$.

Denote by $C_{a}$ a cycle containing exactly once the arrows $a_{i}, i=1, \ldots, p_{r}$ and $b_{j}^{\prime}, b_{j}^{\prime \prime}, j=1, \ldots, q_{r}$, and denote by $C_{b}$ a cycle containing exactly once the arrows $b_{j}, j=1, \ldots, q_{r}$ and $a_{i}^{\prime}, a_{i}^{\prime \prime}, i=1, \ldots, p_{r}$. Then one can check that the irreducible cycles of $Q$ are $C_{a}, C_{b}$, and the $C_{i}$ up to cyclic equivalence. Therefore, we can write

$$
W=\sum_{i=1}^{l} \lambda_{i} C_{i}+\alpha C_{a}+\beta C_{b}+\text { extra terms }
$$

where $\lambda_{i}, \alpha, \beta \in k$ and the extra terms are linear combinations of reducible cycles.

First, we show that we can assume that $\lambda_{i} \neq 0$ for all $i=1, \ldots, l$.

If $p_{r}+q_{r} \geq 3$, then we have immediately $\lambda_{i} \neq 0$ for $i=1, \ldots, l$ by the rigidity of $W$. Assume that $p_{r}=q_{r}=1$. Then $Q$ is of this form:

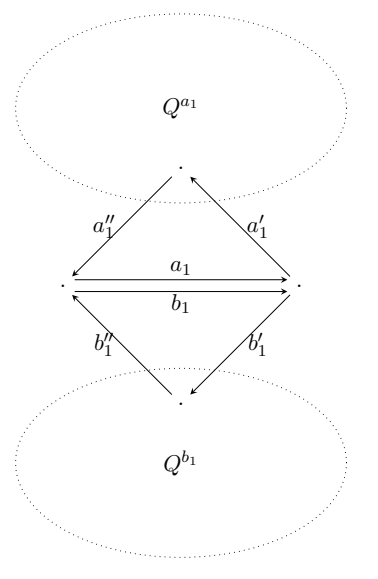

We can assume (up to renumbering) that $C_{1}$ is cyclically equivalent to $a_{1} a_{1}^{\prime} a_{1}^{\prime \prime}$ and that $C_{2}$ is cyclically equivalent to $b_{1} b_{1}^{\prime} b_{1}^{\prime \prime}$, and we have $C_{a}=$ 
$a_{1} b_{1}^{\prime} b_{1}^{\prime \prime}$ and $C_{b}=b_{1} a_{1}^{\prime} a_{1}^{\prime \prime}$. Then it is easy to see that the rigidity of $W$ implies that $\lambda_{i} \neq 0$ for $i=3, \ldots, l$ and that $\lambda_{1} \lambda_{2}-\alpha \beta \neq 0$. Therefore, up to the automorphism of $Q$ exchanging $a_{1}$ and $b_{1}$, we can assume that $\lambda_{1} \lambda_{2} \neq 0$.

Since the potential $W$ is homogeneous of degree 1 , we have

$$
\begin{aligned}
& d\left(a_{i}\right)+d\left(a_{i}^{\prime}\right)+d\left(a_{i}^{\prime \prime}\right)=d\left(b_{j}\right)+d\left(b_{j}^{\prime}\right)+d\left(b_{j}^{\prime \prime}\right)=1 \\
& \quad \text { for all } i=1, \ldots, p_{r}, j=1, \ldots, q_{r} .
\end{aligned}
$$

By definition, we have

$$
\begin{aligned}
& d\left(C_{a}\right)=\sum_{i=1}^{p_{r}} d\left(a_{i}\right)+\sum_{j=1}^{q_{r}}\left(d\left(b_{j}^{\prime}\right)+d\left(b_{j}^{\prime \prime}\right)\right) \quad \text { and } \\
& d\left(C_{b}\right)=\sum_{j=1}^{q_{r}} d\left(b_{j}\right)+\sum_{i=1}^{p_{r}}\left(d\left(a_{i}^{\prime}\right)+d\left(a_{i}^{\prime \prime}\right)\right) .
\end{aligned}
$$

Hence, combining (6.3.1) and (6.3.2), we get

$$
d\left(C_{a}\right)+d\left(C_{b}\right)=p_{r}+q_{r} .
$$

Using (6.3.3) and the fact that $d\left(C_{a}\right)$ and $d\left(C_{b}\right)$ are nonnegative (since $d$ is a map $Q_{1} \rightarrow\{0,1\}$ ), we divide the proof into four subcases.

Case 1: $d\left(C_{a}\right) \geq 2$ and $d\left(C_{b}\right) \geq 2$.

In this case, since $W$ is homogeneous of degree 1 , we have $\alpha=\beta=0$, and there is no extra term in the potential $W$. For $i=1, \ldots, l$, we denote by $c_{i}$ the arrow such that $C_{i}=c_{i}^{\prime \prime} c_{i}^{\prime} c_{i}$. Then we define $\varphi$ on $Q_{1}$ by

$$
\varphi(x)= \begin{cases}\lambda_{i} c_{i} & \text { if } x=c_{i}, \\ x & \text { otherwise }\end{cases}
$$

It is then clear that $\varphi$ is an isomorphism of the graded algebra $(k Q, d)$ sending $W_{Q}$ onto $W$.

Case 2: $d\left(C_{a}\right)=1$ and $d\left(C_{b}\right) \geq 2$.

In this case, since $W$ is homogeneous of degree 1 , we have $\beta=0$, and there is no extra term in the potential $W$. Up to cyclic equivalence and renumbering, we can assume that $C_{1}=b_{1}^{\prime \prime} b_{1}^{\prime} b_{1}$ and that $C_{a}=b_{1}^{\prime \prime} b_{1}^{\prime} C_{a}^{\prime}$. For $i=2, \ldots, l$, we denote by $c_{i}$ the arrow such that $C_{i}=c_{i}^{\prime \prime} c_{i}^{\prime} c_{i}$. Now we define $\varphi$ on $Q_{1}$ by

$$
\varphi(x)= \begin{cases}\lambda_{1} b_{1}+\alpha C_{a}^{\prime} & \text { if } x=b_{1} \\ \lambda_{i} c_{i} & \text { if } x=c_{i}, \text { and } i \geq 2 \\ x & \text { otherwise }\end{cases}
$$


Since $C_{a}=b_{1}^{\prime \prime} b_{1}^{\prime} C_{a}^{\prime}$ and $C_{1}=b_{1}^{\prime \prime} b_{1}^{\prime} b_{1}$ are oriented cycles, the path $C_{a}^{\prime}$ has the same source and the same target as $b_{1}$; thus, $\varphi$ is an algebra morphism. Moreover, since $\lambda_{i} \neq 0, \varphi$ is an isomorphism of the completion $k \hat{Q}$. Now, we have

$$
d\left(C_{a}^{\prime}\right)=d\left(C_{a}\right)-d\left(b_{1}^{\prime}\right)-d\left(b_{1}^{\prime \prime}\right)=1-d\left(b_{1}^{\prime}\right)-d\left(b_{1}^{\prime \prime}\right)=d\left(b_{1}\right) .
$$

Therefore, $\varphi$ is an isomorphism of the graded algebra $(k \hat{Q}, d)$ sending $W_{Q}$ onto $W$.

Case 3: $d\left(C_{a}\right)=1$ and $d\left(C_{b}\right)=1$.

From (6.3.3) we automatically have $p_{r}=q_{r}=1$. All the cycles $C_{1}=$ $a_{1}^{\prime \prime} a_{1}^{\prime} a_{1}, C_{2}=b_{1}^{\prime \prime} b_{1}^{\prime} b_{1}, C_{b}=a_{1}^{\prime \prime} a_{1}^{\prime} b_{1}, C_{a}=b_{1}^{\prime \prime} b_{1}^{\prime} a_{1}, C_{3}, \ldots, C_{l}$ are homogeneous of degree 1 . For $i=3, \ldots, l$, we denote by $c_{i}$ the arrow such that $C_{i}=c_{i}^{\prime \prime} c_{i}^{\prime} c_{i}$. Since $W$ is rigid, we have $\lambda_{1} \lambda_{2}-\alpha \beta \neq 0$. And since the grading $d$ makes $W$ homogeneous of degree 1 , there is no extra term in the potential $W$, and we have $d\left(a_{1}\right)=d\left(b_{1}\right)$. Then we can define

$$
\varphi(x)= \begin{cases}\lambda_{1} a_{1}+\beta b_{1} & \text { if } x=a_{1}, \\ \lambda_{2} b_{1}+\alpha a_{1} & \text { if } x=b_{1}, \\ \lambda_{i} c_{i} & \text { if } x=c_{i}, \text { and } i \geq 3 \\ x & \text { otherwise. }\end{cases}
$$

Since $\lambda_{1} \lambda_{2}-\alpha \beta=0$, this algebra morphism is an isomorphism. Moreover, since $d\left(a_{1}\right)=d\left(b_{1}\right), \varphi:(k Q, d) \rightarrow(k Q, d)$ is an isomorphism of graded algebras. By construction, it sends $W_{Q}$ onto $W$.

Case 4: $d\left(C_{a}\right)=0$.

From (6.3.3), we have $d\left(C_{b}\right) \geq 2$. Hence, since $W$ is homogeneous of degree 1 , we have $\alpha=\beta=0$. In this case, since the degree of $C_{a}$ is 0 , the oriented cycles of the quiver $Q$ which are homogeneous of degree 1 are cyclically equivalent to something of the form $C_{i} C_{a}^{n}$ where $n \in \mathbb{N}$. Thus, we can write up to cyclic equivalence:

$$
W=\sum_{i=1}^{l} C_{i} P_{i}\left(C_{a}\right),
$$

where $P_{i} \in k \llbracket X \rrbracket$ is a power series with constant term $\lambda_{i} \neq 0$. For each $i=1, \ldots, l$, we write $C_{i}=c_{i}^{\prime \prime} c_{i}^{\prime} c_{i}$, and we define

$$
\varphi(x)= \begin{cases}c_{i} P_{i}\left(C_{a}\right) & \text { if } x=c_{i}, \\ x & \text { otherwise }\end{cases}
$$


Then $\varphi$ is an automorphism of the completion $k \hat{Q}$ since $\lambda_{i} \neq 0$ for all $i=$ $1, \ldots, l$. Since $d\left(C_{a}\right)=0$, this automorphism is an automorphism of the graded algebra $(k \hat{Q}, d)$.

The other cases, when

- there exists $1 \leq i \leq p_{r}$ and $1 \leq j \leq q_{r}$ such that $Q^{a_{i}}=\varnothing$ and $Q^{b_{j}}=\varnothing$, and - for all $i=1, \ldots p_{r}$, we have $Q^{a_{i}} \neq \varnothing$, and there exists $j$ such that $Q^{b_{j}}=\varnothing$, are simpler since in these cases there are fewer irreducible cycles. The proof is left to the reader.

REMARK 6.12.

(1) Note that the first part of this lemma can be deduced directly from [DWZ, Theorem 5.7]. Indeed, since $W$ is rigid, the quiver with potential $(Q, W)$ is right equivalent in the sense of [DWZ, Definition 4.2] to $\left(Q, W_{Q}\right)$. (There exists a sequence $s$ such that $\mu_{s}(Q)$ is acyclic; therefore, $\mu_{s}(Q, W)$ is right equivalent to $\mu_{s}\left(Q, W_{Q}\right)$.) By definition, this implies that there exists an automorphism of completed path algebras $\varphi: k \hat{Q} \rightarrow k \hat{Q}$ which is the identity on the vertices, and such that $\varphi\left(W_{Q}\right)$ is cyclically equivalent to $W$. However, we have proved this lemma constructing explicitly the isomorphism $\varphi$.

(2) In the case $p_{r}=q_{r}=1$, it might happen that $\lambda_{1} \lambda_{2}=0$. Then the automorphism $\varphi$ constructed above will exchange the arrows $a_{1}$ and $b_{1}$. The degree map $d^{\prime}$ will satisfy $d^{\prime}\left(a_{1}\right)=d\left(b_{1}\right)$ and $d^{\prime}\left(b_{1}\right)=d\left(a_{1}\right)$. This is the only case where $d$ and $d^{\prime}$ are not the same.

From Lemma 6.10, we deduce the following result, which is a restatement of the second part of Theorem 6.9 and finishes the proof of Theorem 6.9.

Corollary 6.13. Let $\Lambda$ be an algebra of global dimension at most 2 and of cluster type $\widetilde{A}_{p, q}$. Then there exist a quiver $Q \in \mathcal{M}_{p, q}^{\widetilde{A}}$ and a $W_{Q^{-g} \text { grading }}$ $d^{\prime}$ such that $\Lambda$ is isomorphic to the degree 0 part of $\operatorname{Jac}\left(Q, W_{Q}, d\right)$.

Proof. By Proposition 3.11, there exists a reduced graded quiver with potential $(Q, W, d)$, such that $\bar{\Lambda} \underset{\mathbb{Z}}{\sim} \operatorname{Jac}(Q, W, d)$. Moreover, $W$ is rigid, and $d$ is a $W$-grading. By Theorem 6.8, the quiver $Q$ is in $\mathcal{M}_{p, q}^{\widetilde{A}}$. By Lemma 6.10, there exists a $W_{Q}$-grading $d^{\prime}$ such that we have $\operatorname{Jac}(Q, W, d) \underset{\mathbb{Z}}{\sim} \operatorname{Jac}\left(Q, W_{Q}\right.$, $\left.d^{\prime}\right)$. Therefore, $\Lambda$ is isomorphic to the degree 0 part of $\operatorname{Jac}\left(Q, W_{Q}, d^{\prime}\right)$. 
REMARK 6.14.

(1) This corollary implies that an algebra $\Lambda$ of cluster type $\widetilde{A}_{p, q}$ is always isomorphic to an algebra of the form $k Q_{\Lambda} / I$, where the relations are paths of length 2 .

(2) This corollary gives a description of the iterated tilted algebras of global dimension at most 2 of type $\widetilde{A}_{p, q}$. A description of all iterated tilted algebras (not distinguishing with respect to their global dimension) of type $\widetilde{A}_{p, q}$ has been given in $[\mathrm{AmS}$, Theorem (A)].

Corollary 6.15. There are only finitely many algebras (up to Morita equivalence) of global dimension at most 2 and of cluster type $\widetilde{A}_{p, q}$.

Proof. There are only finitely many quivers in the set $\mathcal{M}_{p, q}^{\widetilde{A}}$, and given $Q \in \mathcal{M}_{p, q}^{\widetilde{A}}$, there are finitely many $W_{Q^{-} \text {-gradings. }}$

\subsection{An alternative description of the weight}

In this section, we give an explicit way to compute the weight of an algebra of cluster type $\widetilde{A}_{p, q}$.

Definition 6.16. Let $Q$ be a quiver in $\mathcal{M}_{p, q}^{\tilde{A}}$, and let $d$ be a $\mathbb{Z}$-grading on $Q$ such that the potential $W_{Q}$ is homogeneous of degree 1 . Then let $a_{1}, \ldots, a_{p_{r}}$ be the $p$-arrows, and let $b_{1}, \ldots, b_{q_{r}}$ be the $q$-arrows, of $Q$. The weight of the graded quiver $(Q, d)$ is defined to be

$$
w\left(Q, W_{Q}, d\right):=\sum_{l=1}^{p_{r}} d\left(a_{l}\right)-\sum_{l=1}^{q_{r}} d\left(b_{l}\right) .
$$

The aim of the section is to show the following.

Proposition 6.17. Let $Q \in \mathcal{M}_{p, q}^{\widetilde{A}}$, and let $d$ be a $W_{Q}$ grading. Let $\Lambda$ be the degree 0 part of the graded algebra $\operatorname{Jac}\left(Q, W_{Q}, d\right)$. Then $\Lambda$ is an algebra of global dimension at most 2 and of cluster type $\widetilde{A}_{p, q}$, and we have

$$
w(\Lambda)=w\left(Q, W_{Q}, d\right)
$$

Proof. The first part of the statement follows from Theorem 6.9.

Let $s$ be a sequence of mutation such that $\mu_{s}\left(Q, W_{Q}\right)=(H, 0)$, where $Q_{H}$ is an acyclic quiver of type $\widetilde{A}_{p, q}$. Define a grading $\partial$ on $Q_{H}$ by $\mu_{s}^{\mathrm{L}}\left(Q, W_{Q}\right.$, $d)=(H, 0, \partial)$. By definition, we have $w(\Lambda)=w(H, 0, \partial)$. Hence, the proof of the proposition comes directly from the following technical lemma. 
Lemma 6.18. Let $Q$ be a quiver in $\mathcal{M}_{p, q}^{\widetilde{A}}$, and let $d$ be a $\mathbb{Z}$-grading on $Q$ such that $W_{Q}$ is homogeneous of degree 1 . Let $i$ be a vertex of $Q$. Then we have

$$
w\left(\mu_{i}^{\mathrm{L}}\left(Q, W_{Q}, d\right)\right)=w\left(Q, W_{Q}, d\right)
$$

Proof. We define the grading $d^{\prime}$ on the quiver $\mu_{i}(Q)$ by $\mu_{i}^{\mathrm{L}}(Q, W, d)=$ $\left(\mu_{i}(Q, W), d^{\prime}\right)$. We distinguish the following different cases.

Case 1: There exists $\alpha$ in the nonoriented cycle $C$ of $Q$ such that $i \in Q^{\alpha}$ and $i \neq u(\alpha)$.

Then the vertices adjacent to $i$ are not in the nonoriented cycle. Therefore, the mutation of $Q$ at $i$ does not affect the nonoriented cycle, so the weight clearly remains the same.

Case 2: $i=u(\alpha)$ for some arrow $\alpha$ which is on the nonoriented cycle $C$.

Assume that $\alpha$ is a $p$-arrow. There exists a 3-cycle $\alpha^{\prime \prime} \alpha^{\prime} \alpha$ which is a summand of $W$.
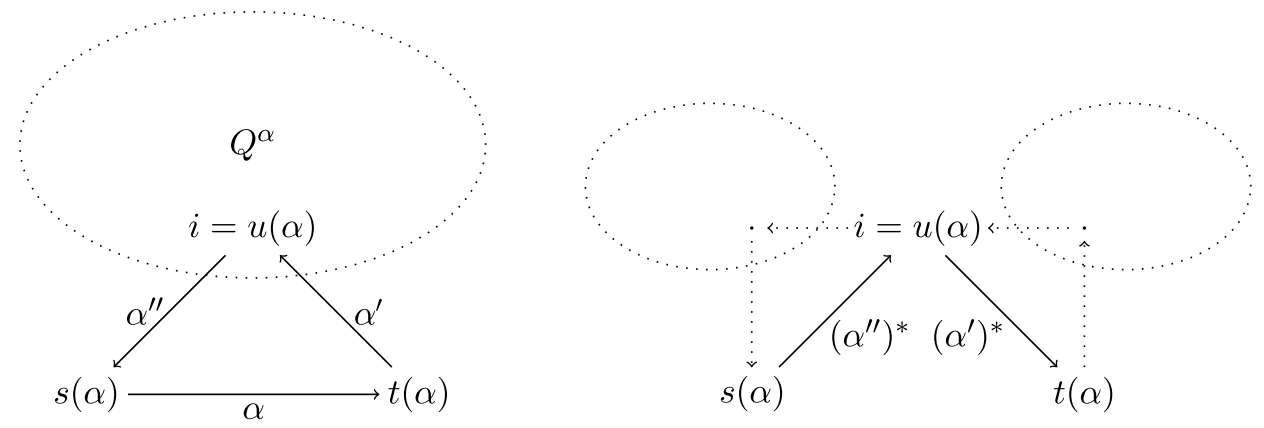

The new arrows $\left(\alpha^{\prime}\right)^{*}$ and $\left(\alpha^{\prime \prime}\right)^{*}$ become $p$-arrows in the nonoriented cycle. Therefore, we have

$$
\begin{aligned}
w\left(\mu_{i}^{\mathrm{L}}(Q, W, d)\right) & =w(Q, W, d)-d(\alpha)+d^{\prime}\left(\left(\alpha^{\prime}\right)^{*}\right)+d^{\prime}\left(\left(\alpha^{\prime \prime}\right)^{*}\right) \\
& =w(Q, W, d)-d(\alpha)-d\left(\alpha^{\prime}\right)-d\left(\alpha^{\prime \prime}\right)+1 \\
& =w(Q, W, d)
\end{aligned}
$$

The last equality holds since $\alpha^{\prime \prime} \alpha^{\prime} \alpha$ is a summand in the potential $W$, and hence we have $d(\alpha)+d\left(\alpha^{\prime}\right)+d\left(\alpha^{\prime \prime}\right)=1$.

Case 3: $i$ is on the nonoriented cycle $C$ between two p-arrows.

Then the quiver $Q$ locally looks like 

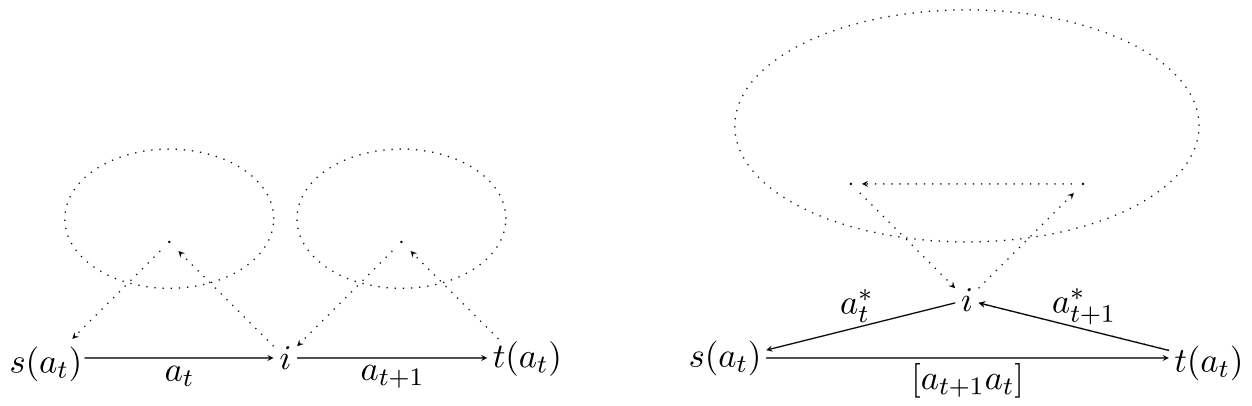

The arrows $a_{t}$ and $a_{t+1}$ are replaced by the new $p$-arrow $\left[a_{t+1} a_{t}\right]$ in the nonoriented cycle. Hence, we have

$$
\begin{aligned}
w\left(\mu_{i}^{\mathrm{L}}(Q, W, d)\right) & =w(Q, W, d)-d\left(a_{t}\right)-d\left(a_{t+1}\right)+d^{\prime}\left(\left[a_{t+1} a_{t}\right]\right) \\
& =w(Q, W, d) .
\end{aligned}
$$

The case where $i$ is between two $q$ arrows is similar.

Case $4: i$ is a sink of the nonoriented cycle $C$.

Assume that $i$ is the target of the $p$-arrow $a_{l}$ and the target of the $q$-arrow $b_{t}$.
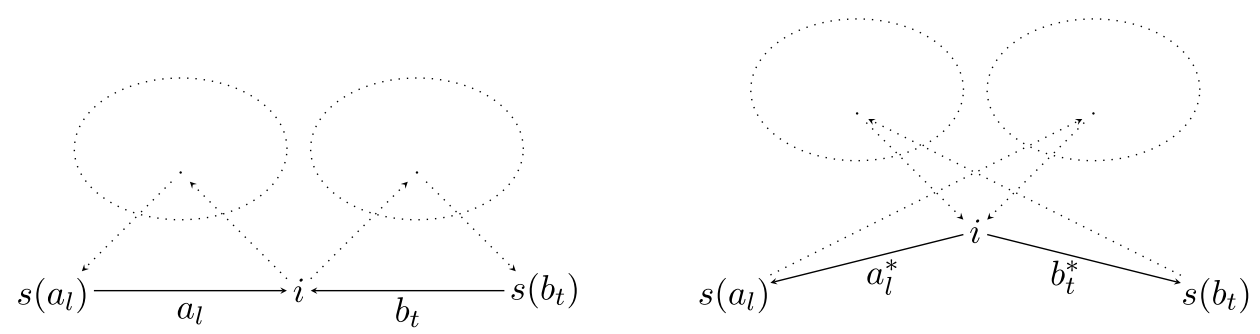

Then the arrows $a_{l}$ and $b_{t}$ are replaced by the arrows $a_{l}^{*}$ and $b_{t}^{*}$, and we have

$$
\begin{aligned}
w\left(\mu_{i}^{\mathrm{L}}(Q, W, d)\right) & =w(Q, W, d)-d\left(a_{l}\right)+d\left(b_{t}\right)+d^{\prime}\left(b_{t}^{*}\right)-d^{\prime}\left(a_{l}^{*}\right) \\
& =w(Q, W, d)-d\left(a_{l}\right)+d\left(b_{t}\right)+\left(-d\left(b_{t}\right)+1\right)-\left(-d\left(a_{l}\right)+1\right) \\
& =w(Q, W, d) .
\end{aligned}
$$

The case where $i$ is the source of one $p$-arrow and of one $q$-arrow is similar. 
This alternative description of the weight gives us the following consequences.

Corollary 6.19. The number of derived equivalence classes of algebras of cluster type $\widetilde{A}_{p, q}$ is $[p / 2]+[q / 2]+1$ if $p \neq q$ and $[p / 2]+1$ if $p=q$.

Proof. Let $Q$ be a quiver in $\mathcal{M}_{p, q}^{\widetilde{A}}$. It is clear from the definition that $w$ is maximal when the $W_{Q}$-grading satisfies $d\left(a_{l}\right)=1$ for $l=1, \ldots, p_{r}$ and $d\left(b_{j}\right)=0$ for $j=1, \ldots, q_{r}$. In this case, the weight is equal to $p_{r}=p-$

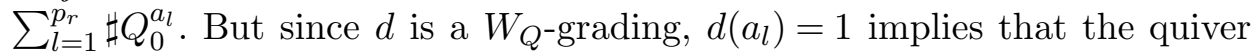
$Q^{a_{l}}$ is nonempty. Then we have $w=p_{r}=p-\sum_{l=1}^{p_{r}} \sharp Q_{0}^{a_{l}} \leq p-p_{r}$. Thus, we have $w \leq[p / 2]$. For the same reason, we have $w \geq-[q / 2]$. Now it is easy to see that all values $-[q / 2],-[q / 2]+1, \ldots,[p / 2]$ can occur.

COROLlary 6.20. Let $\Lambda$ be an algebra of cluster type $\widetilde{A}_{p, q}$, which is not piecewise hereditary. Then there exists a tilting object $T$ in $\mathcal{D}^{b}(\Lambda)$ such that the quiver of $\operatorname{End}_{\mathcal{D}^{b}(\Lambda)}(T)$ has an oriented cycle.

Proof. Let $-q / 2 \leq w \leq p / 2$ be a nonzero integer. We construct an algebra $B$ of cluster type $\widetilde{A}_{p, q}$ of weight $w$ such that $Q_{B}$ has an oriented cycle. Without loss of generality, we can assume that $w>0$. We define $B$ as follows:

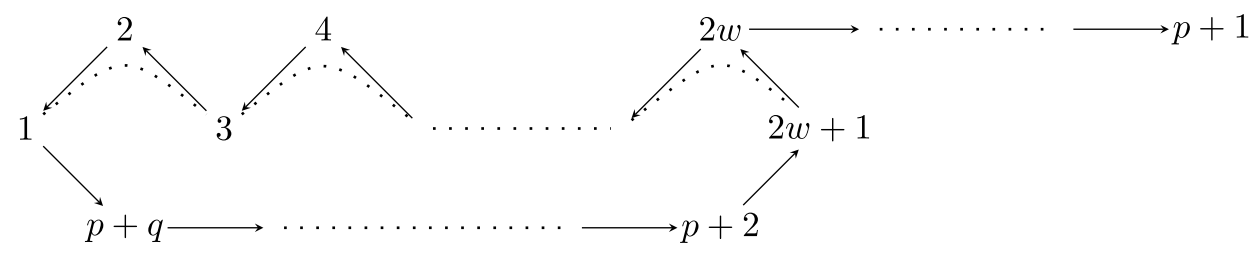

It is clear that $B$ is the degree 0 part of the Jacobian algebra $\operatorname{Jac}\left(Q, W_{Q}, d\right)$ with the graded quiver

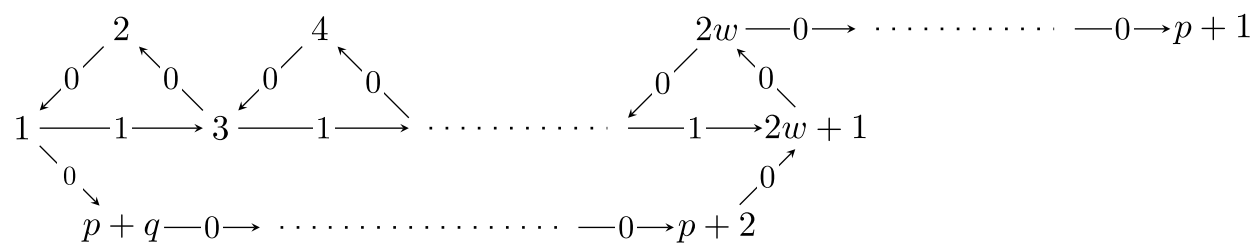

Then by Theorem 4.5 , this algebra $B$ is the endomorphism algebra of some tilting complex $T \in \mathcal{D}^{b}(\Lambda)$. 
Corollary 6.21. Let $\Lambda$ be an algebra of cluster type $\widetilde{A}_{p, q}$ and of weight $w$; then the Coxeter polynomial of $\Lambda$ is

$$
X^{p+q}-(-1)^{w} X^{p-w}-(-1)^{w} X^{q+w}+1 .
$$

Proof. By definition, the Coxeter matrix is the matrix of the automorphism $\tau$ at the level of the Grothendieck group $K_{0}\left(\mathcal{D}^{b}(\Lambda)\right)$ in the basis $\left\{\left[S_{i}\right] \mid 1 \leq i \leq p+q\right\}$ consisting of the representatives of the simples. The Coxeter polynomial $C(X)$ is its characteristic polynomial.

The result is already known for $w=0$. Without loss of generality, we can assume that $0<w \leq p / 2$. By the above results, we can assume that $\Lambda$ is given by the following quiver with relations:

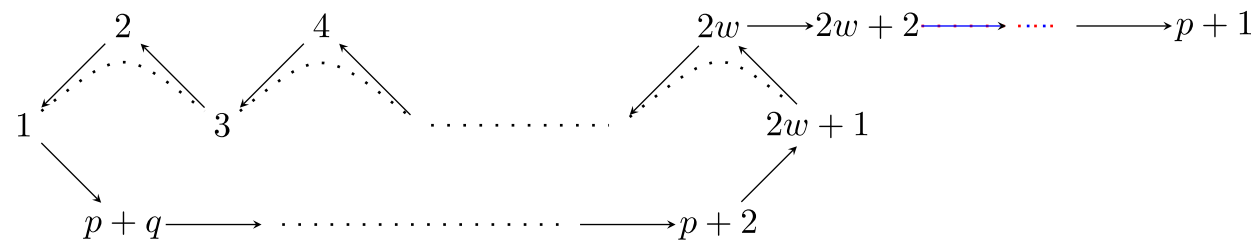

Then for $0 \leq j \leq w-1$, the projective resolution of $S_{2 j+1}$ is given by

$$
0 \longrightarrow P_{2 j+3} \longrightarrow P_{2 j+2} \longrightarrow P_{2 j+1} \longrightarrow S_{2 j+1} \longrightarrow 0 \text {. }
$$

Thus, one easily checks that,

$$
\begin{aligned}
& \text { for } 0 \leq j \leq w-1, \quad \tau\left[S_{2 j+1}\right]=\left[S_{2 j+3}[1]\right]=-\left[S_{2 j+3}\right] \\
& \text { in } K_{0}\left(\mathcal{D}^{b}(\Lambda)\right) .
\end{aligned}
$$

We also have the projective resolutions

$$
\begin{gathered}
0 \longrightarrow P_{p+2} \longrightarrow P_{2 w+1} \longrightarrow S_{2 w+1} \longrightarrow 0 \quad \text { and } \\
\text { for } p+2 \leq j \leq p+q, \quad 0 \longrightarrow P_{j+1} \longrightarrow P_{j} \longrightarrow S_{j} \longrightarrow 0
\end{gathered}
$$

where we use the convention $p+q+1=1$. Hence, we have

$$
\begin{gathered}
\tau\left[S_{2 w+1}\right]=\left[S_{p+2}\right] \quad \text { and, } \\
\text { for } p+2 \leq j \leq p+q, \quad \tau\left[S_{j}\right]=\left[S_{j+1}\right] \quad \text { in } K_{0}\left(\mathcal{D}^{b}(\Lambda)\right) .
\end{gathered}
$$


Combining (6.4.1) and (6.4.2), we get

$$
\tau^{q+w}\left[S_{1}\right]=(-1)^{w} \tau^{q}\left[S_{2 w+1}\right]=(-1)^{w}\left[S_{1}\right] .
$$

Similarly, we have

$$
\text { for } 2 w+3 \leq j \leq p+1, \quad \tau\left[S_{j}\right]=\left[S_{j-1}\right] \quad \text { in } K_{0}\left(\mathcal{D}^{b}(\Lambda)\right) .
$$

Now we have to separate the case where $w=1$. Assume that $w \geq 2$; then we have

$$
\tau\left[S_{2 w+2}\right]=\left[P_{2 w-2}\right] \quad \text { in } K_{0}\left(\mathcal{D}^{b}(\Lambda)\right),
$$

(6.4.5) for $2 \leq j \leq w-1, \quad \tau\left[P_{2 j}\right]=-\left[I_{2 j}\right]=-\left[P_{2 j-2}\right] \quad$ in $K_{0}\left(\mathcal{D}^{b}(\Lambda)\right)$.

Hence, if $p+1 \geq 2 w+2$, then we have $I_{2} \cong P_{p+1}$, and we get the following equalities in $K_{0}\left(\mathcal{D}^{b}(\Lambda)\right)$ :

$$
\begin{aligned}
\tau^{p-w}\left[P_{2}\right] & =-\tau^{p-w-1}\left[P_{p+1}\right]=\tau^{p-w+2}\left[S_{p+1}\right] \\
& =\tau^{w-1} \tau^{p-2 w-1}\left[S_{p+1}\right]=\tau^{w-1}\left[S_{2 w+2}\right] \quad \text { by }(6.4 .3) \\
& =\tau^{w-2}\left[P_{2 w-2}\right] \quad \text { by }(6.4 .4) \\
& =(-1)^{w}\left[P_{2}\right] \quad \text { by }(6.4 .5) .
\end{aligned}
$$

If $p+1=2 w+1$, we have $I_{2} \cong P_{2 w}$, and we also get $\tau^{p-w}\left[P_{2}\right]=(-1)^{w}\left[P_{2}\right]$.

Assume that $w$ is odd. Then one can checks that the set

$$
\begin{gathered}
\left\{\left[S_{2 j+1}\right], 0 \leq j \leq w\right\} \cup\left\{\left[S_{j}\right], 2 w+2 \leq j \leq p+q\right\} \\
\cup\left\{\left[P_{2 j}\right], 1 \leq j \leq w-1\right\} \cup\left\{\left[I_{2}\right]\right\}
\end{gathered}
$$

is a basis of $K_{0}\left(\mathcal{D}^{b}(\Lambda)\right)$. Therefore, the Coxeter matrix is diagonalizable, and $C(X)=\left(X^{q+w}+1\right)\left(X^{p-w}+1\right)$.

Assume that $w$ is even. Then the set

$$
\left\{\left[S_{2 j+1}\right], 0 \leq j \leq w\right\} \cup\left\{\left[S_{j}\right], 2 w+2 \leq j \leq p+q\right\} \cup\left\{\left[P_{2 j}\right], 1 \leq j \leq w-1\right\}
$$

is linearly independent in $K_{0}\left(\mathcal{D}^{b}(\Lambda)\right)$, and we have the relation

$$
\left[I_{2}\right]-\sum_{j=2 w+2}^{p+1}\left[S_{j}\right]-\sum_{j=1}^{w-1}(-1)^{j}\left[P_{2 j}\right]=\sum_{j=p+2}^{p+q}\left[S_{j}\right]+\sum_{j=1}^{w}(-1)^{j}\left[S_{2 j+1}\right] .
$$


This element is an eigenvector of the eigenvalue 1. Hence,

$$
\frac{\left(X^{q+w}-1\right)\left(X^{p-w}-1\right)}{(X-1)}
$$

divides the Coxeter polynomial $C(X)$. Since the degree of $C(X)$ is $p+q$, and since we know that both the leading coefficient and the absolute term of $C(X)$ are 1 , it follows that

$$
C(X)=\frac{\left(X^{q+w}-1\right)\left(X^{p-w}-1\right)}{(X-1)} \cdot(X-1)=\left(X^{q+w}-1\right)\left(X^{p-w}-1\right) .
$$

For the case $w=1$, we introduce the following notations for $4 \leq j \leq p$ :

$$
\begin{aligned}
M_{j} & :=\operatorname{Ker}\left(I_{2} \rightarrow I_{j+1} \oplus I_{j+1}\right) \cong \operatorname{Coker}\left(P_{2} \rightarrow P_{j} \oplus P_{j}\right), \\
M_{3} & :=\operatorname{Ker}\left(I_{2} \rightarrow I_{4} \oplus I_{4}\right) \cong P_{2}, \\
M_{p+1} & :=\operatorname{Coker}\left(P_{2} \rightarrow P_{p+1} \oplus P_{p+1}\right) \cong I_{2} .
\end{aligned}
$$

Then we have,

$$
\text { for } 4 \leq j \leq p+1, \quad \tau\left[M_{j}\right]=\left[M_{j-1}\right] .
$$

Therefore, we get the equalities in $K_{0}\left(\mathcal{D}^{b}(\Lambda)\right)$ :

$$
\tau^{p-1}\left[P_{2}\right]=-\tau^{p-2}\left[I_{2}\right]=\tau^{p-2}\left[M_{p+1}\right]=-\left[M_{3}\right]=-\left[P_{2}\right] .
$$

Finally, it is easy to see that the set

$$
\left\{\left[M_{j}\right], 3 \leq j \leq p+1\right\} \cup\left\{\left[S_{1}\right],\left[S_{3}\right]\right\} \cup\left\{\left[S_{j}\right], p+2 \leq j \leq p+q\right\}
$$

is a basis of $\mathbb{Q} \otimes_{\mathbb{Z}} K_{0}\left(\mathcal{D}^{b}(\Lambda)\right)$. This finishes the proof.

We end this section by giving a result linking the weight of an algebra of cluster type $\widetilde{A}_{p, q}$ with the set of cluster-tilting objects of $\mathcal{C}_{\widetilde{A}_{p, q}}$ coming from tilting complexes in $\mathcal{D}^{b}(\Lambda)$. We start with some notation.

Let $\Lambda$ be an algebra of global dimension at most 2 which is $\tau_{2}$-finite. We define a subset $\mathcal{T}_{\Lambda}$ of the set of tilting complexes of $\mathcal{D}^{b}(\Lambda)$ by

$$
\mathcal{T}_{\Lambda}:=\left\{T \in \mathcal{D}^{b}(\Lambda) \text { tilting } \mid \operatorname{gldim}\left(\operatorname{End}_{\mathcal{D}^{b}(\Lambda)}(T)\right) \leq 2\right\} .
$$

By Theorem 2.4, the set $\pi_{\Lambda}\left(\mathcal{T}_{\Lambda}\right) \subset \mathcal{C}_{\Lambda}$ is a subset of the set of cluster-tilting objects of $\mathcal{C}_{\Lambda}$. Moreover, if $\Lambda_{1}$ and $\Lambda_{2}$ are derived equivalent, they are cluster equivalent, and we clearly have $\pi_{\Lambda_{1}}\left(\mathcal{T}_{\Lambda_{1}}\right)=\pi_{\Lambda_{2}}\left(\mathcal{T}_{\Lambda_{2}}\right)$. We prove here the converse in the case of algebras of cluster type $\widetilde{A}_{p, q}$, and we compare the sets $\pi_{\Lambda_{1}}\left(\mathcal{T}_{\Lambda_{1}}\right)$ and $\pi_{\Lambda_{2}}\left(\mathcal{T}_{\Lambda_{2}}\right)$ when $w\left(\Lambda_{1}\right) \neq w\left(\Lambda_{2}\right)$. 
Proposition 6.22. Let $\Lambda_{1}$ and $\Lambda_{2}$ be two algebras of global dimension 2 and of cluster type $\widetilde{A}_{p, q}$. Then we have

- $0 \leq w\left(\Lambda_{1}\right)<w\left(\Lambda_{2}\right) \Rightarrow \pi_{2}\left(\mathcal{T}_{\Lambda_{2}}\right) \subsetneq \pi_{1}\left(\mathcal{T}_{\Lambda_{1}}\right)$,

- $w\left(\Lambda_{2}\right)<w\left(\Lambda_{1}\right) \leq 0 \Rightarrow \pi_{2}\left(\mathcal{T}_{\Lambda_{2}}\right) \subsetneq \pi_{1}\left(\mathcal{T}_{\Lambda_{1}}\right)$

- $w\left(\Lambda_{1}\right) w\left(\Lambda_{2}\right)<0 \Rightarrow \pi_{1}\left(\mathcal{T}_{\Lambda_{1}}\right) \backslash \pi_{2}\left(\mathcal{T}_{\Lambda_{2}}\right) \neq \emptyset \neq \pi_{2}\left(\mathcal{T}_{\Lambda_{2}}\right) \backslash \pi_{1}\left(\mathcal{T}_{\Lambda_{1}}\right)$

Proof. We first show the inclusion in the first claim. Without loss of generality, we can assume that $w\left(\Lambda_{2}\right)=w\left(\Lambda_{1}\right)+1>0$. It is enough to show that $\pi_{2}\left(\Lambda_{2}\right) \in \pi_{1}\left(\mathcal{T}_{\Lambda_{1}}\right)$. We denote by $H$ some hereditary algebra of type $\widetilde{A}_{p, q}$, and we denote by $\pi_{2}$ a triangle functor $\pi_{2}: \mathcal{D}^{b}\left(\Lambda_{2}\right) \rightarrow \mathcal{C}_{H}$.

Let $\left(Q, W_{Q}, d\right)$ be a graded quiver with potential such that we have isomorphisms of $\mathbb{Z}$-graded algebras $\operatorname{End}_{\mathcal{C}_{H}}\left(\pi_{2} \Lambda_{2}\right) \cong \operatorname{Jac}\left(Q, W_{Q}, d\right)$. Since $w\left(\Lambda_{2}\right) \geq 1$, there exists a $p$-arrow $a_{i} \in Q$ such that $d\left(a_{i}\right)=1$. Since $d$ is

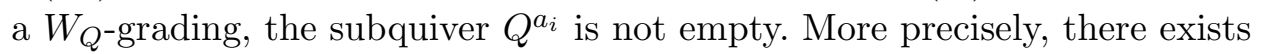
arrows $a_{i}^{\prime}$ and $a_{i}^{\prime \prime}$ such that $a_{i} a_{i}^{\prime} a_{i}^{\prime \prime}$ is an oriented triangle in $Q$.

Define a new degree $d^{\prime}$ on $Q$ by

$$
d^{\prime}(x)= \begin{cases}0 & \text { if } x=a_{i} \\ 1 & \text { if } x=a_{i}^{\prime} \\ d(x) & \text { otherwise }\end{cases}
$$

It is immediate to see that $d^{\prime}$ is a $W_{Q}$-grading. Define the algebra $\Lambda_{3}$ as the degree 0 part of the graded Jacobian algebra $\operatorname{Jac}\left(Q, W_{Q}, d^{\prime}\right)$. By Theorem 6.9 , it is an algebra of global dimension 2 which is of cluster type $\widetilde{A}_{p, q}$, and by Corollary 3.2 we can assume that $\pi_{2}\left(\Lambda_{2}\right)=\pi_{3}\left(\Lambda_{3}\right)$, where $\pi_{3}$ is a triangle functor $\pi_{3}: \mathcal{D}^{b}\left(\Lambda_{3}\right) \rightarrow \mathcal{C}_{\Lambda_{3}}$. Moreover, by Proposition 6.17 , we have $w\left(\Lambda_{3}\right)=w\left(\Lambda_{2}\right)-1=w\left(\Lambda_{1}\right)$. Therefore, by Theorem 4.5 , the algebra $\Lambda_{3}$ is derived equivalent to $\Lambda_{1}$. The image of $\Lambda_{3}$ through this equivalence is clearly an object $X$ in $\mathcal{T}_{\Lambda_{1}}$ which satisfies $\pi_{1}(X) \cong \pi_{2}\left(\Lambda_{2}\right)$.

For any $0 \leq w \leq[p / 2]$, one can easily construct a quiver $Q$ such that $Q$ admits a $W_{Q}$-grading of weight $w$ but no $W_{Q}$-grading of weight $w+1$. Therefore, the inclusion is strict.

The second point holds by symmetry.

For the third point, it is enough to see that for any $w>0$, the quiver $Q$ constructed in the proof of Corollary 6.20 satisfies the following: there exists a $W_{Q}$-grading of weight $w$, and for any $w^{\prime}<0$ there is no $W_{Q}$-grading of weight $w^{\prime}$. The argument for $w<0$ holds by symmetry.

We end this section by asking the following intriguing questions. 


\section{Question 6.23.}

- Let $\Lambda_{1}$ and $\Lambda_{2}$ be $\tau_{2}$-finite algebras of global dimension at most 2 which are cluster equivalent. Do we have the implication

$$
\pi_{1}\left(\mathcal{T}_{\Lambda_{1}}\right)=\pi_{2}\left(\mathcal{T}_{\Lambda_{2}}\right) \Rightarrow \mathcal{D}^{b}\left(\Lambda_{1}\right) \cong \mathcal{D}^{b}\left(\Lambda_{2}\right) ?
$$

- Let $\Lambda$ be a $\tau_{2}$-finite algebra of global dimension at most 2 . Does the following implication hold?

$$
\pi_{\Lambda}\left(\mathcal{T}_{\Lambda}\right)=\left\{X \in \mathcal{C}_{\Lambda} \mid X \text { cluster-tilting }\right\} \Rightarrow \Lambda \text { piecewise hereditary }
$$

\subsection{Example}

In this section we compute explicitly all basic algebras (up to isomorphism) of global dimension at most 2 and of cluster type $\widetilde{A}_{2,2}$ and organize them according to their derived equivalence classes.

The strategy consists of first describing all quivers (up to isomorphism of quivers) which are in the set $\mathcal{M}_{2,2}^{\widetilde{A}}$. In our case, an easy computation or [K1] shows that there are only four different quivers in $\mathcal{M}_{2,2}^{\widetilde{A}}$ :
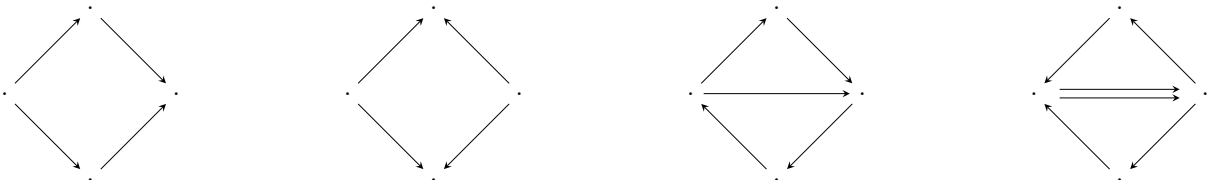

Note that since $p=q$, there is an isomorphism between the two quivers corresponding to $p_{r}=2, q_{r}=1$ and $p_{r}=1, q_{r}=2$ :
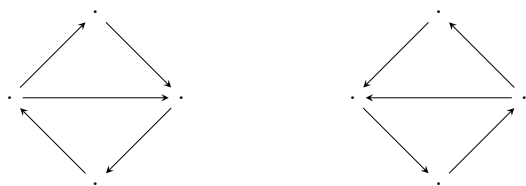

Then one can easily check that there are 11 graded quivers $(Q, d)$ with $Q \in$ $\mathcal{M}_{p, q}^{\widetilde{A}}$ and $d$ a $W_{Q}$-grading up to isomorphism of graded quiver. Therefore, there are exactly 11 nonisomorphic algebras of global dimension 2 and of cluster type $\widetilde{A}_{2,2}$.

The only possible weights are $|w|=0$ or $|w|=1$. Therefore, these 11 algebras are divided into two derived equivalence classes. 
There are eight algebras of global dimension at most 2 which are derived equivalent to $\widetilde{\widetilde{A}}_{2,2}$ :
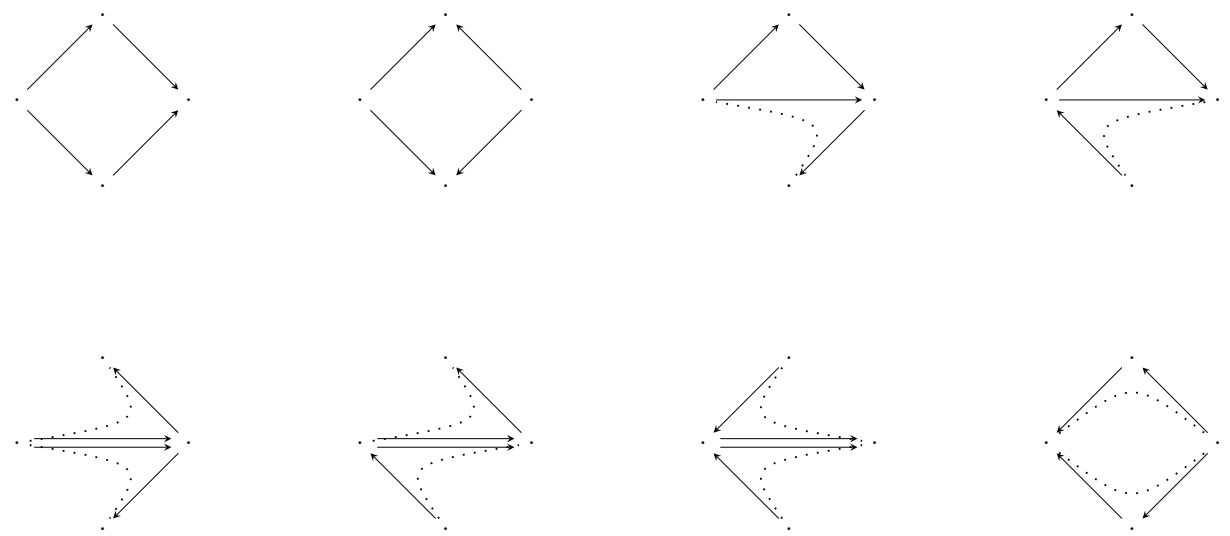

There are three algebras of global dimension at most 2 which are of cluster type $\widetilde{A}_{2,2}$ and are not derived equivalent to $\widetilde{A}_{2,2}$. They are all derived equivalent to each other and not piecewise hereditary:
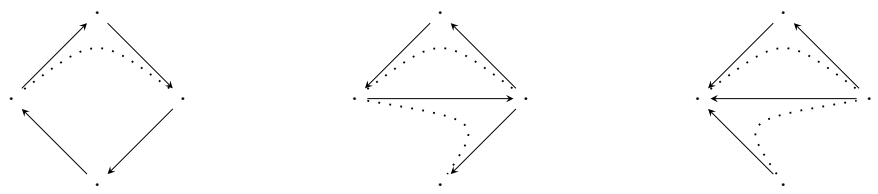

Acknowledgment. The authors would like to thank one of the referees for valuable comments and for pointing us out a mistake in a previous version of this article.

\section{REFERENCES}

[A] C. Amiot, Cluster categories for algebras of global dimension 2 and quivers with potential, Ann. Inst. Fourier (Grenoble) 59 (2009), 2525-2590. MR 2640929.

[AO1] C. Amiot and S. Oppermann, Cluster equivalence and graded derived equivalence, arXiv:math.RT/1003.4916 [math.RT]

[AO2] - The image of the derived category in the cluster category, Int. Math. Res. Not. IMRN 2013, 733-760. DOI 10.1093/imrn/rns010.

[Am] I. Assem, Tilted algebras of type $A_{n}$, Comm. Algebra 10 (1982), 2121-2139. MR 0675347. DOI 10.1080/00927878208822826.

[AmS] I. Assem and A. Skowroński, Iterated tilted algebras of type $\tilde{A}_{n}$, Math. Z. 195 (1987), 269-290. MR 0892057. DOI 10.1007/BF01166463. 
[Ba] J. Bastian, Mutation classes of $\tilde{A}_{n}$-quivers and derived equivalence classification of cluster-tilted algebras of type $\tilde{A}_{n}$, Algebra Number Theory 5 (2011), 567-594. MR 2889747. DOI 10.2140/ant.2011.5.567.

[BGP] I. N. Bernšteĭn, I. M. Gel'fand, and V. A. Ponomarev, Coxeter functors, and Gabriel's theorem, Uspehi Mat. Nauk 28 (1973), no. 2, 19-33; English translation in Russian Math. Surveys 28 (1973), no. 2, 17-32. MR 0393065.

[BIRS1] A. B. Buan, O. Iyama, I. Reiten, and J. Scott, Cluster structures for 2-CalabiYau categories and unipotent groups, Compos. Math. 145 (2009), no. 4, 10351079. MR 2521253. DOI 10.1112/S0010437X09003960.

[BIRS2] A. B. Buan, O. Iyama, I. Reiten, and D. Smith, Mutation of cluster-tilting objects and potentials, Amer. J. Math. 133 (2011), 835-887. MR 2823864. DOI 10.1353/ajm.2011.0031.

[BMRRT] A. B. Buan, R. Marsh, M. Reineke, I. Reiten, and G. Todorov, Tilting theory and cluster combinatorics, Adv. Math. 204 (2006), 572-618. MR 2249625. DOI 10.1016/j.aim.2005.06.003.

[BV] A. B. Buan and D. F. Vatne, Derived equivalence classification for clustertilted algebras of type $A_{n}$, J. Algebra 319 (2008), 2723-2738. MR 2397404. DOI 10.1016/j.jalgebra.2008.01.007.

[DWZ] H. Derksen, J. Weyman, and A. Zelevinsky, Quivers with potentials and their representations, I: Mutations, Selecta Math. (N.S.) 14 (2008), 59-119. MR 2480710. DOI 10.1007/s00029-008-0057-9.

[FZ] S. Fomin and A. Zelevinsky, Cluster algebras, I: Foundations, J. Amer. Math. Soc. 15 (2002), 497-529. MR 1887642. DOI 10.1090/S0894-0347-01-00385-X.

[H] D. Happel, On the derived category of a finite-dimensional algebra, Comment. Math. Helv. 62 (1987), 339-389. MR 0910167. DOI 10.1007/BF02564452.

[HU] D. Happel and L. Unger, "On the set of tilting objects in hereditary categories" in Representations of Algebras and Related Topics, Fields Inst. Commun. 45, Amer. Math. Soc., Providence, 2005, 141-159. MR 2146246.

[Hub] A. Hubery, The cluster complex of an hereditary Artin algebra, Algebr. Represent. Theory 14 (2011), 1163-1185. MR 2844758. DOI 10.1007/ s10468-010-9229-3.

[IY] O. Iyama and Y. Yoshino, Mutation in triangulated categories and rigid Cohen-Macaulay modules, Invent. Math. 172 (2008), 117-168. MR 2385669. DOI 10.1007/s00222-007-0096-4.

[K1] B. Keller, Quiver mutation in Java, Java applet, http://www.math.jussieu .fr/ keller/quivermutation/.

[K2] , On triangulated orbit categories, Doc. Math. 10 (2005), 551-581. MR 2184464.

[K3] - Deformed Calabi-Yau completions, with appendix by Michel Van den Bergh, J. Reine Angew. Math. 654 (2011), 125-180. MR 2795754. DOI 10. 1515/CRELLE.2011.031.

[KR] B. Keller and I. Reiten, Acyclic Calabi-Yau categories, with an appendix by Michel Van den Bergh, Compos. Math. 144 (2008), 1332-1348. MR 2457529. DOI 10.1112/S0010437X08003540.

[KY] B. Keller and D. Yang, Derived equivalences from mutations of quivers with potential, Adv. Math. 226 (2011), 2118-2168. MR 2739775. DOI 10.1016/j. aim.2010.09.019. 
[V] D. Vossieck, The algebras with discrete derived category, J. Algebra 243 (2001), 168-176. MR 1851659. DOI 10.1006/jabr.2001.8783.

Claire Amiot

Institut Fourier-UMR 5582

100 rue des maths

38402 Saint Martin d'Hères

France

Claire.Amiot@ujf-grenoble.fr

Steffen Oppermann

Institutt for matematiske fag

Norwegian University of Science and Technology

7491 Trondheim

Norway

steffen.oppermann@math.ntnu.no 